\title{
Principles of carbon nanotube dielectrophoresis
}

\author{
Wenshan $\mathrm{Li}^{1,3, \dagger}$, Frank Hennrich ${ }^{1,2,4}$, Benjamin S. Flavel ${ }^{1}$, Simone Dehm ${ }^{1}$, Manfred Kappes ${ }^{1,2}$, and \\ Ralph Krupke ${ }^{1,3,4}(\bowtie)$ \\ ${ }^{1}$ Institute of Nanotechnology, Karlsruhe Institute of Technology, Karlsruhe 76021, Germany
${ }^{2}$ Institute of Physical Chemistry, Karlsruhe Institute of Technology, Karlsruhe 76021, Germany
${ }^{3}$ Institute of Materials Science, Technische Universität Darmstadt, Darmstadt 64287, Germany
${ }^{4}$ Institute for Quantum Materials and Technologies, Karlsruhe Institute of Technology, Karlsruhe 76021, Germany
${ }^{+}$Present address: School of Mechanical Engineering, Shanghai Jiao Tong University, No. 800 Dongchuan Road, Shanghai 200240, China
}

(C) The Author(s) 2021, corrected publication 2021

Received: 6 July 2020 / Revised: 12 October 2020 / Accepted: 15 October 2020

\section{ABSTRACT}

Dielectrophoresis (DEP) describes the motion of suspended objects when exposed to an inhomogeneous electric field. It has been successful as a method for parallel and site-selective assembling of nanotubes from a dispersion into a sophisticated device architecture. Researchers have conducted extensive works to understand the DEP of nanotubes in aqueous ionic surfactant solutions. However, only recently, DEP was applied to polymer-wrapped single-walled carbon nanotubes (SWCNTs) in organic solvents due to the availability of ultra-pure SWCNT content. In this paper, the focus is on the difference between the DEP in aqueous and organic solutions. It starts with an introduction into the DEP of carbon nanotubes (CNT-DEP) to provide a comprehensive, in-depth theoretical background before discussing in detail the experimental procedures and conditions. For academic interests, this work focuses on the CNT-DEP deposition scheme, discusses the importance of the electrical double layer, and employs finite element simulations to optimize CNT-DEP deposition condition with respect to the experimental observation. An important outcome is an understanding of why DEP in organic solvents allows for the deposition and alignment of SWCNTs in low-frequency and even static electric fields, and why the response of semiconducting SWCNTs (s-SWCNTs) is strongly enhanced in non-conducting, weakly polarizable media. Strategies to further improve CNT-DEP for s-SWCNT-relevant applications are given as well. Overall, this work should serve as a practical guideline to select the appropriate setting for effective CNT DEPs.
\end{abstract}

\section{KEYWORDS}

single-walled carbon nanotubes, hydrodynamics, assembly, deposition, alignment, polarizability, dielectrophoresis

\section{Introduction}

The chirality (denoted with one set of indices $(n, m)$ )-resolved band structure of single-walled carbon nanotubes (SWCNTs) enables many exceptional electrical and optical properties $[1,2]$, making nanotubes a competitive candidate-material for novel electronics [3-6] and optoelectronics [7, 8]. Indeed, many benchmarks of SWCNT applications have been reached, such as 40-nm-footprint transistors [9], 5-nm gate length field-effect transistors (FETs) [10], 3-dimensional computation-storagein-stack chips [11], submicron-gated ultrahigh radio-frequency electronics $[4,12,13]$ and room-temperature single-photon emitter [4]. However, there is still no approved technique for the reliable integration of SWCNTs into complex device architectures to meet with different applications. This technology gap needs to be closed to promote reproducibility of carbon nanotube (CNT) devices.

The fundamental challenge of SWCNT integration is to precisely and site-selectively assemble nanotubes to achieve the largest possible packing density with a negligible inter-tube dielectric screening effect [14-16]. Perfectly aligned CNTs with an equidistant pitch are ideal to ensure the optimum of device performance and reproducibility. So far, methods aiming at this purpose can be categorized as two approaches: in-situ on-substrate horizontal growth of CNT parallel arrays and solution-based CNT post-assembling. Methods based on CNT on-substrate growth have yielded nanotube films with a highdegree of alignment and a packing density of up to 70 tubes/ $\mu \mathrm{m}$ [17-22]. However, due to an inefficient growth-control over CNT chirality in this way, metallic (m-) SWCNTs, to some extent, are always present in devices, significantly suppressing device performance. Comparatively, solution-based post-assembly methods, such as shear-force guided SWCNT alignment [23, $24]$ is known to lack fine-control over CNT orientations and locations. The Langmuir-Schaefer method $[25,26]$, a widely used technique for fabricating molecular thin films, illustrates inefficiency in assembling monolayer SWCNT arrays, thus detrimental to transistor performances because of the increased inter-tube electrostatic screening effect in multilayer assemblies. Recently, transistors based on monolayer CNT films were fabricated through a dose-controlled, floating evaporative selfassembly method [12, 13, 27, 28]. The CNT packing density can range from 60 up to 200 tubes/ $\mu \mathrm{m}$, and corresponding devices have demonstrated promising saturated on-state currents which exceed their silicon-based counterparts [28, 29]. This improvement, beyond the state-of-the-art, is attributed to the removal of polymer residues through high-temperature annealing. More precise pitch scaling of CNT arrays is 
demonstrated through template-guided CNT assembly using DNA bricks [30-33]. However, the performance of transistors formed in this way is still inferior for electrical applications $[30,31,33]$. Comparatively, high CNT packing densities have also been achieved using dielectrophoresis (DEP) [15, 34], which is a method versatile for selective deposition of micro-/nanoparticles $[35,36]$. The advantage of DEP for nanotube integration is its capability to obtain the site-selective deposition of CNTs at a monolayer coverage, a high degree of alignment, and a high packing density. Unlike electrophoresis, DEP does not require depositing particles to be charged [37] but makes use of their induced dipole moments within an external inhomogeneous electric field. Beneficially, the placement and density of particle depositions are tunable and controllable by simply optimizing electrode structures or adjusting electric fields [38, 39].

DEP was first developed in the 1950s [40] and then applied to biological applications in the 1970s [41]. Since 2000, the method has seen a revival due to the successful manipulation of nanoparticles [35, 42-44]. In 2003, DEP had been used for CNT depositions aiming at selectively separating CNT bundles [45] and m-SWCNTs [38] out of aqueous solutions using $\mathrm{MHz}$ alternating current (AC) electric fields. Later on, a study of the frequency dependence of CNT DEPs [46] illustrated that semiconducting (s-) SWCNTs could deposit from water-based suspensions at sub- $\mathrm{MHz}$ frequency because of the ionicsurfactant induced surface conductance. Strategically, using low-frequency DEP in combination with highly-purified s-SWCNT aqueous dispersions eventually realized a highperformance CNT electronics as discussed in Ref. [4]. However, the presence of residual metallic nanotube content still obstructs CNT aqueous dispersions [15, 34, 46, 47]. By contrast, this problem is significantly suppressed through dispersing SWCNTs into organic solvents (such as toluene) with polymer wrapping [34, 48-50].

In order to realize ultra-pure s-SWCNT depositions through DEP, the deposition scheme of this technique must be revisited, which is the motivation of this work. We discuss both theoretical and experimental aspects of CNT-DEP with organic solvents and compare the results to those of aqueous solutions. Thereby, we obtain a comprehensive view of the principles of CNT DEP. Beyond the conventional scope of discussing merely the dielectric response and electric fields, both thermal and fluidic physics involved in the DEP is also included here. The framework of this study is organized in the following way: Section 2 reviews the theory about CNT motions and solvent fluidics during the CNT-DEP process. Section 3 focuses on the CNTDEP deposition scheme and discusses the importance of understanding the formation of the electrical double layer (EDL). Then in Section 4, finite element simulations are performed to investigate the optimum DEP condition for the deposition of s-SWCNTs and compared with experimental observations. Finally, Section 5 summarizes the discussions.

\section{Theoretical consideration}

\subsection{Motion of CNT in suspension}

The DEP deposition for a SWCNT is driven by the nonuniform local electric field which is generated through electrically biased nano- or microscale electrodes. The electric field induces a dipole moment mainly along the longitudinal axis of the nanotube. Because of the nonuniformity of the electric field, a net DEP force ensues which moves the nanotube inwards (positive DEP) or outwards (negative DEP) into the deposition region with respect to the direction of the electric-field gradient. In practice, there are many other factors such as the gravitational force and Brownian motion, also playing an essential role in the motion of SWCNTs in liquids. In order to quantitatively describe the movement of a SWCNT within DEP, one must consider all these factors and analyze their effects on either the translational or the rotational CNT motions.

\subsubsection{Translational motion}

In a fluid system, the ratio of the inertial force to the viscous force, known as the Reynolds number, is less than 0.02 for a suspended CNT with length $\leq 100 \mu \mathrm{m}$ as discussed in Refs. $[35,51]$. The small Reynolds number indicates that the inertial force of a SWCNT moving in the fluid is negligible compared to its viscous force, meaning that the motion of the nanotube follows Stoke's law [35].

The translational motion of a CNT driven by a force $\vec{F}$ within the fluid is expressed as

$$
m_{\mathrm{CNT}} \frac{\mathrm{d} \vec{u}_{\mathrm{CNT}}}{\mathrm{d} t}=-\gamma\left(\vec{u}_{\mathrm{CNT}}-\vec{v}\right)+\vec{F}
$$

where $m_{\mathrm{CNT}}$ is the CNT mass. $\vec{u}_{\mathrm{CNT}}$ and $\vec{v}$ denote the velocity of the CNT and the fluid, respectively. The term $-\gamma\left(\vec{u}_{\mathrm{CNT}}-\vec{v}\right)$ represents the drag force of this nanotube experienced in the fluid, with the friction factor $\gamma$ given by $\gamma=3 \pi \eta l_{\mathrm{CNT}} / \ln \left(2 l_{\mathrm{CNT}} /\right.$ $\left.d_{\mathrm{CNT}}\right)$. $l_{\mathrm{CNT}}$ and $d_{\mathrm{CNT}}$ are the CNT length and diameter, respectively, and $\eta$ stands for the dynamic viscosity of the fluid [52].

For a given force $\vec{F}$ and a fluid velocity $\vec{v}$, the CNT instant velocity is

$$
\vec{u}_{\mathrm{CNT}}=\left(\vec{u}_{0, \mathrm{CNT}}-\vec{v}-\frac{\vec{F}}{\gamma}\right) \exp ^{-(\gamma / m) t}+\vec{v}+\frac{\vec{F}}{\gamma}
$$

$\vec{u}_{0, \mathrm{CNT}}$ indicates the initial velocity of the CNT. The characteristic time for the initial acceleration is $\tau=m_{\mathrm{CNT}} / \gamma$. Within this work, $\tau$ is calculated based on the parameters displayed in Table 1. Notably, $\tau$ is below $10^{-11}$ seconds for a CNT with $1 \mu \mathrm{m}$ in length and $2.5 \mathrm{~nm}$ in diameter. Figures $1(\mathrm{a})$ and $1(\mathrm{~b})$ show the dependence of $\tau$ on the chiral index $(n, m)$ of the nanotube dispersed either in water $(\eta=0.894 \mathrm{mPa} \cdot \mathrm{s}$, also see the Table 1$)$ or in toluene $(\eta=0.583 \mathrm{mPa} \cdot \mathrm{s}$, Table 1$)$. For a practical timescale larger than $\tau$, it is reliable to assume that the CNT is moving at a terminal velocity of

$$
\vec{u}_{\mathrm{CNT}}=\vec{v}+\frac{\vec{F}}{\gamma}
$$

In practice, both the acceleration of fluid and diffusion of fluid vorticity also influence the CNT motion, making the situation more complicated. However, that discussion goes beyond the scope of this work.

\section{(a) Gravitational force}

The mass density of a $(n, m)$ SWCNT is $\rho_{\mathrm{CNT}}=3.89 \times 10^{4} /$ $\sqrt{n^{2}+m^{2}+m n} \mathrm{~kg} / \mathrm{m}^{3}[53,54]$. Based on this expression, the gravitational force for a SWCNT in a fluid with a density of $\rho_{\mathrm{m}}$ is

$$
\vec{F}_{\mathrm{g}}=v\left(\rho_{\mathrm{CNT}}-\rho_{\mathrm{m}}\right) \vec{g}
$$

where $\vec{g}$ is the gravitational acceleration and $v=\pi l_{\mathrm{CNT}} d_{\mathrm{CNT}}^{2} / 6$ is the CNT volume. Based on Eq. (3), the terminal velocity of the SWCNT driven by its gravity is

$$
\vec{u}_{\mathrm{g}}=\frac{v\left|\rho_{\mathrm{CNT}}-\rho_{\mathrm{m}}\right| \vec{g}}{\gamma}=\frac{d_{\mathrm{CNT}}^{2} \ln \left(2 l_{\mathrm{CNT}} / d_{\mathrm{CNT}}\right)\left|\rho_{\mathrm{CNT}}-\rho_{\mathrm{m}}\right| \vec{g}}{18 \eta}
$$

Figures 1(c) and 1(d) illustrate the terminal velocities induced 
Table 1 Variables, parameters and referenced values

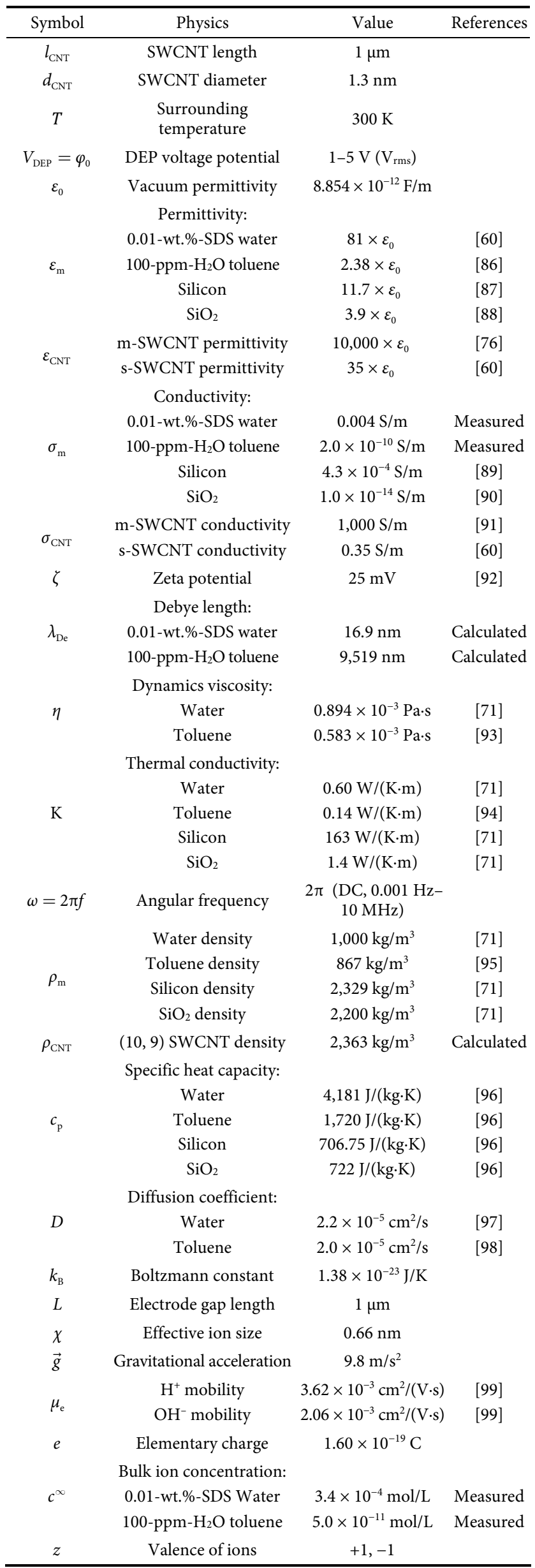
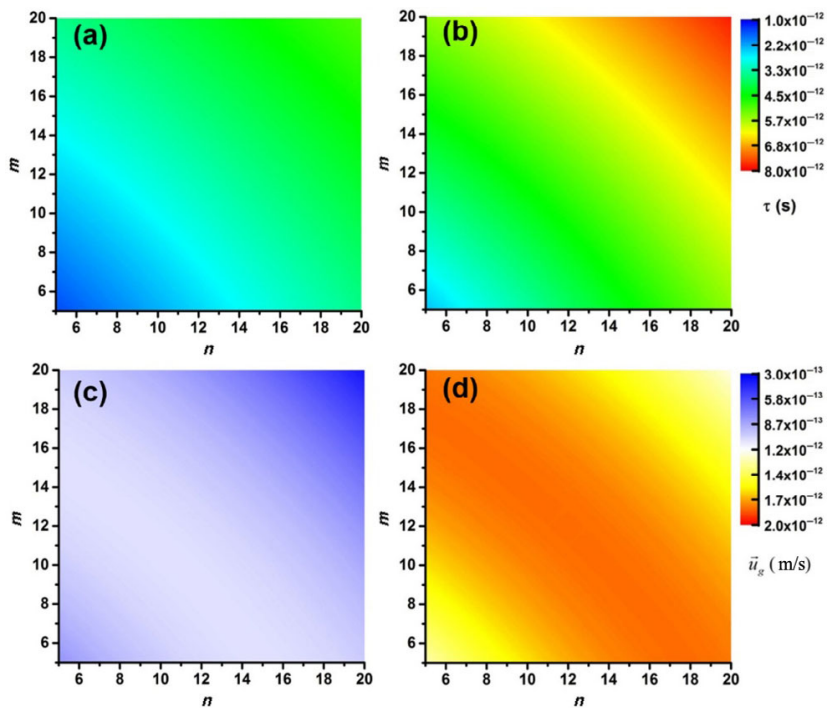

Figure 1 ((a) and (b)) Characteristic acceleration time scale $\tau$ and ((c), (d)) terminal velocity induced by the gravitational force for CNTs dispersed in ((a), (c)) water and ((b), (d)) toluene with $l_{\mathrm{CNT}}=1 \mu \mathrm{m}$. The data is plotted versus the chiral index $n$ and $m$.

by the gravitational force for a $1-\mu \mathrm{m}$-long SWCNT which is dispersed either in (a) water $\left(\rho_{\mathrm{m}}=1.0 \times 10^{3} \mathrm{~kg} / \mathrm{m}^{3}\right)$ or in (b) toluene $\left(\rho_{\mathrm{m}}=866.9 \mathrm{~kg} / \mathrm{m}^{3}\right)$, respectively. In both cases, this velocity is below $10^{-11} \mathrm{~m} / \mathrm{s}$, and negligible compared to those induced through Brownian motion and DEP as discussed below.

\section{(b) Brownian motion}

The Brownian motion describes the random motion of a suspending particle originating from its collisions with atoms or molecules in fluids or gases [55]. Statistically, the random displacement of a solution-based CNT arising from the Brownian motion follows a Gaussian profile as $[56,57]$

$$
\Delta L=\sqrt{2 D t}
$$

here $\Delta L$ is the displacement of the CNT in the liquid driven by the Brownian motion, and $D$ stands for the diffusion tensor of the nanotube in this liquid. $t$ is the time of observation.

A CNT is a prolate spheroid. In order to obtain an analytical solution in the Eq. (6), two translational hydrodynamic friction factors $\gamma_{\mathrm{par}}$ and $\gamma_{\mathrm{per}}$ are needed to describe both motions in parallel and perpendicular directions with respect to the CNT longitudinal axis [58]. Therefore, the displacement of the CNT becomes [56]

$$
D_{\text {trans }}=\frac{k_{\mathrm{B}} T}{6 \pi \eta} \frac{2 \ln \left(l_{\mathrm{CNT}} / d_{\mathrm{CNT}}\right)-\gamma_{\mathrm{par}}-\gamma_{\mathrm{per}}}{l_{\mathrm{CNT}}}
$$

Figure 2(a) shows the translational diffusion for a CNT with a diameter of $1.3 \mathrm{~nm}$ as a function of length. For a $1 \mu \mathrm{m}$ long CNT dispersed in either water or in toluene, $D_{\text {trans }}$ is $3.1 \mu \mathrm{m}^{2} / \mathrm{s}$ or $5.0 \mu \mathrm{m}^{2} / \mathrm{s}$, respectively. The Brownian motion gives rise to several orders of magnitude larger CNT displacement than that induced by the gravitational force. Thus, one can anticipate that an effective DEP deposition only occurs if the DEP translational displacement of the CNT is larger than the Brownian-motion one.

\section{(c) Dielectrophoretic force}

Based on Pohl's theory, for a particle of prolate-spheroid shape [37], the DEP force $\vec{F}_{\text {DEP }}$ is

$$
\vec{F}_{\mathrm{DEP}}=(\vec{p} \cdot \nabla) \vec{E}
$$

where $\vec{p}$ is the induced dipole moment of the particle within 

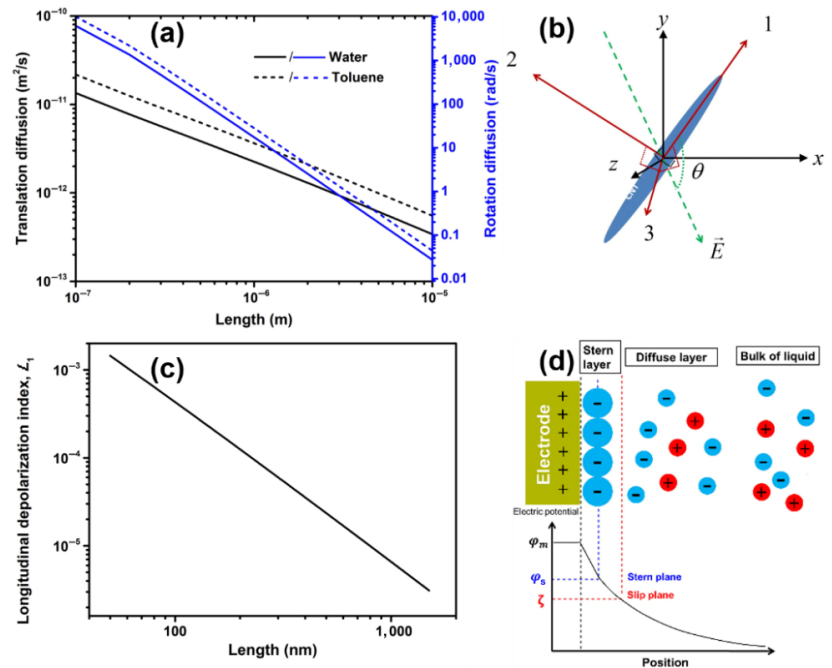

Figure 2 (a) Translational and rotational Brownian diffusion of CNTs dispersed in water and toluene as a function of CNT length. $d_{\mathrm{CNT}}=1.3 \mathrm{~nm}$. (b) Schematic defining the coordinate system in a DEP experiment. (c) Length dependence of the longitudinal (tube-axis parallel) depolarization index of CNTs with a diameter of $1.3 \mathrm{~nm}$. (d) Schematic diagram of the Stern modified Gouy-Chapman electrical double layer and corresponding electrical potential distribution.

the electric field $\vec{E}$. In formula,

$$
\vec{p}=v \tilde{\alpha} \vec{E}
$$

here $\alpha$ stands for the complex effective polarizability of this particle.

Figure 2(b) illustrates the schematic of a typical DEP experiment for a prolate-ellipsoid particle, such as a CNT. In order to define both directions of the electric field and nanotube, two groups of three orthogonal axes are employed as shown in this figure. $x, y, z$ are picked to define the direction of the electric field, and 1, 2, 3 axes chosen the radii axis $\mathrm{a}_{1}, \mathrm{a}_{2}$, $\mathrm{a}_{3}$ of the CNT, respectively. The angle $\theta$ represents the relative orientation between the electric field and the long axis 1 of the nanotube. Based on these definitions, the effective polarizability of a CNT with respect to each principal axis $\mathbb{N}(\mathbb{N}=1,2,3)$ is [52]

$$
\tilde{\alpha}_{\mathbb{N}}=\varepsilon_{\mathrm{m}} \tilde{f}_{\mathrm{CM}, \mathbb{N}}
$$

with

$$
\tilde{f}_{\mathrm{CM}, \mathbb{N}}=\frac{\tilde{\varepsilon}_{\mathrm{cnt}}-\tilde{\varepsilon}_{\mathrm{m}}}{\left(\tilde{\varepsilon}_{\mathrm{m}}+\left(\tilde{\varepsilon}_{\mathrm{cnt}}-\tilde{\varepsilon}_{\mathrm{m}}\right) L_{\mathbb{N}}\right)}, \tilde{\varepsilon}=\varepsilon-i \frac{\sigma}{\omega}
$$

here, $\tilde{\varepsilon}_{\mathrm{CNT}}, \tilde{\varepsilon}_{\mathrm{m}}$ stand for complex dielectric permittivities of the nanotube and medium. $\varepsilon$ and $\sigma$ indicate their real permittivity and conductivity, respectively. $\omega$ is the angular frequency of the electric field. Parameter $\tilde{f}_{\mathrm{CM}, \mathbb{N}}$ is frequencydependent and known as the Clausius-Mossotti factor (CMF) $[46,59,60]$. The depolarization index $L_{\mathbb{N}}$ is determined by the CNT geometry, as discussed in Ref. [61]. In general, the CNT length $l_{\mathrm{CNT}}$ is much larger than the width $d_{\mathrm{CNT}}$, which gives rise to the $L_{2}$ and $L_{3}$, making $L_{2}=L_{3} \approx 1 / 2$, while significantly suppresses the component $L_{1}=d_{\mathrm{CNT}}^{2} / l_{\mathrm{CNT}}^{2}\left[\ln \left(2 l_{\mathrm{CNT}} / d_{\mathrm{CNT}}\right)-\right.$ $1] \ll 1$ (as shown in Fig. 2(c)). Therefore, for a SWCNT with a large aspect ratio, it is applicable to consider only the DEP force arising from the nanotube's long-axis polarizability [62]. In the time-averaging,

$$
\begin{aligned}
\left\langle\vec{F}_{\mathrm{DEP}}\right\rangle= & \frac{1}{2} \mathfrak{R}\left[(\vec{p} \cdot \nabla) \vec{E}^{*}\right]=\frac{1}{4} v \Re\left[\tilde{\alpha}_{1}\right] \nabla|\vec{E}|^{2} \\
& -\frac{1}{2} v \operatorname{Im}\left[\tilde{\alpha}_{1}\right](\nabla \times(\mathfrak{R}[\vec{E}] \times \operatorname{Im}[\vec{E}]))
\end{aligned}
$$

where ${ }^{\star}$ indicates the complex conjugation. $\mathfrak{R}$ and Im mean to take the real and imaginary parts of variables into concern. The first term $1 / 4 v \Re\left[\tilde{\alpha}_{1}\right] \nabla|\vec{E}|^{2}$ is non-zero for a spatially varying electric field, while the second term $-1 / 2 v \operatorname{Im}\left[\tilde{\alpha}_{1}\right](\nabla \times(\mathfrak{R}[\vec{E}] \times$ $\operatorname{Im}[\vec{E}]))$ is non-zero only if there is a spatially varying phase within DEP circuits which is frequently accessible in travelling wave DEP or electrorotation [52]. Thus, the second term is negligible for a two-microscale-electrode based DEP system at low frequencies. Therefore, the spatial phase is a constant in DEPs involved in this work, leading to

$$
\left\langle\vec{F}_{\mathrm{DEP}}\right\rangle=\frac{\pi d_{\mathrm{CNT}}^{2} l_{\mathrm{CNT}}}{12} \varepsilon_{\mathrm{m}} \mathfrak{R}\left[\frac{\tilde{\varepsilon}_{\mathrm{cnt}}-\tilde{\varepsilon}_{\mathrm{m}}}{\left(\tilde{\varepsilon}_{\mathrm{m}}+\left(\tilde{\varepsilon}_{\mathrm{CNT}}-\tilde{\varepsilon}_{\mathrm{m}}\right) L_{1}\right)}\right] \nabla\left|\vec{E}_{\mathrm{rms}}\right|^{2}
$$

with $\vec{E}_{\mathrm{rms}}$ standing for the root mean square of the electric field $\vec{E}$.

In practice, the dipole approximation used in Eq. (13) is inaccurate when the CNT is close to a null field or within a region where field strength varies greatly, such as at the edge of electrodes. Under that circumstance, a higher-order moment (such as quadrupole, octupole, etc.) and a corresponding force term become significant and non-negligible.

The expression in the square bracket of Eq. (13) is known as CMF. In general, it has a high-frequency-limit form of

$$
\lim _{\omega \rightarrow \infty} \tilde{f}_{\mathrm{CM}}=\frac{\varepsilon_{\mathrm{cnt}}-\varepsilon_{\mathrm{m}}}{\varepsilon_{\mathrm{m}}}
$$

and low-frequency-limit form of

$$
\lim _{\omega \rightarrow 0} \tilde{f}_{\mathrm{CM}}=\frac{\sigma_{\mathrm{cnt}}-\sigma_{\mathrm{m}}}{\sigma_{\mathrm{m}}}
$$

with considering the depolarization factor $L_{1}$ is on the order of only $10^{-5}$ for a $1-\mu \mathrm{m}$ long nanotube (as shown in Fig. 2(c)).

Recently, Li et al. [60] reported that the low-frequency approximation of the CMF as given in the Eq. (15) is inaccurate when the high-conductivity aqueous surfactant medium $\left(\sigma_{\mathrm{m}} \approx\right.$ $0.004 \mathrm{~S} / \mathrm{m}$ for $0.01 \mathrm{w}-\%$ sodium dodecyl sulfate (SDS)-water) is replaced by a low conductivity solvent, such as toluene $\left(\sigma_{\mathrm{m}} \leq 10^{-11} \mathrm{~S} / \mathrm{m}\right)$ in the CNT DEP. In the latter case, the influence of the depolarization index $L_{1}$ on the magnitude of $\tilde{f}_{\mathrm{CM}}$ increases as the conductivity of the medium drops. Thus, for toluene, the low-frequency limit of the CMF becomes only CNT geometry dependent, and Eq. (15) turns into

$$
\lim _{\omega \rightarrow 0} \tilde{f}_{\mathrm{CM}}=\frac{\sigma_{\mathrm{CNT}}}{\sigma_{\mathrm{CNT}} L_{1}}=\frac{1}{L_{1}}=\frac{l_{\mathrm{CNT}}^{2}}{d_{\mathrm{CNT}}^{2}\left[\ln \left(\frac{2 l_{\mathrm{CNT}}}{d_{\mathrm{CNT}}}\right)-1\right]}
$$

\subsubsection{Rotational motion}

\section{(a) Brownian motion}

Aside from the translational displacement, a SWCNT also rotates in the liquid because of the Brownian motion. A diffusion coefficient $\gamma_{r}$ characterizes such rotational movement. According to the Broersma theory [57], the rotational diffusion tensor $D_{\text {rot }}$ of a prolate ellipsoid is

$$
D_{\text {rot }}=\frac{3 k_{\mathrm{B}} T}{\pi \eta} \frac{\ln \left(l_{\mathrm{CNT}} / d_{\mathrm{CNT}}\right)-\gamma_{r}}{l_{\mathrm{CNT}}^{3}}
$$

In terms of a CNT ( $1 \mu \mathrm{m}$ in length, $1.3 \mathrm{~nm}$ in diameter), the $D_{\text {rot }}$ are $24.3 \mathrm{rad} / \mathrm{s}$ and $39.3 \mathrm{rad} / \mathrm{s}$ in water and in toluene, respectively (as shown in Fig. 2(a)). Thus, in order to realize 
well-aligned CNT deposition, this rotational diffusion needs to be overcome by a sufficient field-induced torque as in DEP.

\section{(b) DEP torque}

The expression of DEP torque $\vec{T}_{\mathrm{DEP}}$ of a particle is [37]

$$
\vec{T}_{\mathrm{DEP}}=\vec{p} \times \vec{E}
$$

Assuming the direction of an electric field is within the 1-2 plane (as shown in Fig. 2(b)), meaning $\vec{E}_{\mathrm{rms}, 3}=0$, thereby $\left\langle T_{\mathrm{DEP}}\right\rangle_{1}=\left\langle T_{\mathrm{DEP}}\right\rangle_{2} \equiv 0$ and the time-averaged dielectrophoretic torque of a CNT is [63]

$$
\left\langle T_{\mathrm{DEP}}\right\rangle_{3}=\frac{\pi d_{\mathrm{CNT}}^{2} l_{\mathrm{CNT}}}{6}\left(L_{2}-L_{1}\right) \vec{E}_{\mathrm{rms}, 1} \vec{E}_{\mathrm{rms}, 2} \varepsilon_{\mathrm{m}} \mathfrak{R}\left[\tilde{f}_{\mathrm{CM}, 1} \cdot \tilde{f}_{\mathrm{CM}, 2}\right]
$$

with $\vec{E}_{\mathrm{rms}, 1}=\vec{E}_{\mathrm{rms}} \cos \theta$ and $\vec{E}_{\mathrm{rms}, 2}=\vec{E}_{\mathrm{rms}} \sin \theta$. Integrating Eq. (11) into the above equation, one can derive

$$
\begin{aligned}
\left\langle T_{\mathrm{DEP}}\right\rangle_{3}= & \frac{\pi d_{\mathrm{CNT}}^{2} l_{\mathrm{CNT}}}{6}\left|\vec{E}_{\mathrm{rms}}\right|^{2} \varepsilon_{\mathrm{m}} \mathfrak{R}\left[\frac{\tilde{\varepsilon}_{\mathrm{cnt}}-\tilde{\varepsilon}_{\mathrm{m}}}{\left(\tilde{\varepsilon}_{\mathrm{m}}+\left(\tilde{\varepsilon}_{\mathrm{CNT}}-\tilde{\varepsilon}_{\mathrm{m}}\right) L_{1}\right)}\right. \\
& \left.-\frac{\tilde{\varepsilon}_{\mathrm{CNT}}-\tilde{\varepsilon}_{\mathrm{m}}}{\left(\tilde{\varepsilon}_{\mathrm{m}}+\left(\tilde{\varepsilon}_{\mathrm{CNT}}-\tilde{\varepsilon}_{\mathrm{m}}\right) L_{2}\right)}\right] \sin \theta \cos \theta
\end{aligned}
$$

\section{(c) CNT alignment}

Based on the torque $T_{\text {DEP }}$ in the Eq. (20), the rotational energy of a CNT is given by

$$
U_{\mathrm{ROT}}=\int\left\langle T_{\mathrm{DEP}}\right\rangle_{\mathrm{CNT}} \mathrm{d} \theta
$$

Concerning the thermal energy $k_{\mathrm{B}} T$, with $k_{\mathrm{B}}$ denoting the Boltzmann constant and $T$ the temperature, determining the Brownian motion, $U_{\mathrm{ROT}}$ follows the Boltzmann distribution using

$$
f(\theta) \mathrm{d} \Omega=\frac{\exp \left(-U_{\mathrm{ROT}} / k_{\mathrm{B}} T\right) \mathrm{d} \Omega}{\int_{0}^{\pi} \exp \left(-U_{\mathrm{ROT}} / k_{\mathrm{B}} T\right) \mathrm{d} \Omega}
$$

with $\mathrm{d} \Omega=2 \pi \sin \theta \mathrm{d} \theta$.

In general, the SWCNT alignment is frequently characterized by the Nematic order parameter $S$, which is zero in a disordered phase and non-zero in an ordered phase as discussed in Refs. $[60,62]$. Assuming that the CNT is aligned within the same plane (such as 1-2 plane in Fig. 2(b)) of the external field, the expression of the Nematic order parameter is

$$
S_{2 \mathrm{~d}}=\int_{-\pi / 2}^{\pi / 2} \cos 2 \theta \cdot f\left(\theta, U_{\mathrm{ROT}}\right) \mathrm{d} \Omega
$$

\subsubsection{Trajectory of motion}

Based on the above discussions, the translational and rotational motions of a CNTs in DEP are $[64,65]$

$$
\begin{gathered}
\vec{u}_{\mathrm{CNT}}=\vec{v}+\frac{\vec{F}_{\mathrm{DEP}}+\vec{F}_{\mathrm{g}}}{\gamma}+\frac{\Delta R_{\mathrm{trans}}}{\Delta t} \\
\varpi_{\mathrm{CNT}}=\varpi_{\mathrm{m}}+\frac{\vec{T}_{\mathrm{DEP}}}{\gamma_{\theta}}+\frac{\Delta R_{\mathrm{rot}}}{\Delta t}
\end{gathered}
$$

with $\Delta R_{\text {trans }}$ and $\Delta R_{\text {rot }}$ standing for the translational and rotational displacements of a CNT induced by the Brownian motion within a time interval of $\Delta t$, respectively, and $\gamma_{\theta}$ defined as

$$
\gamma_{\theta}=\frac{2}{3} \pi \eta \frac{\left(l_{\mathrm{CNT}}^{2}+d_{\mathrm{CNT}}^{2}\right) l_{\mathrm{CNT}}}{2 \ln \left(2 l_{\mathrm{CNT}} / d_{\mathrm{CNT}}\right)-1}
$$

here it is assumed that the fluid velocity $\vec{v}$, and the angular rotation velocity $\varpi_{\mathrm{m}}$ are constants. However, this assumption could be inappropriate to describe the entire motion of a SWCNT in solution when large electric fields are applied in $\mathrm{DEP}$, as discussed in Section 4.

\subsection{Electrohydrodynamic effects}

With the increase of the electric field, the Joule heating becomes significant in DEP. This phenomenon gives rise to an electrothermal force (ETF) on the liquid because of the temperature-induced variations in the conductivity and permittivity of the solvent [66]. As the Joule heating becomes non-negligible, one must consider the effect of buoyancy force on the movement of CNT in DEP. Besides, depending on the electrode geometry, an applied alternating current (AC) DEP bias yields a tangential component at the interface between the electrode and electrolyte. This component points along the electrical double layer (EDL), as shown in Fig. 2(d), contributing to a fluid motion denoted as the AC electroosmotic (ACEO) flow [52]. Usually, this flow, which is field frequency-dependent, leads to an unwanted migration of CNTs. Thereby it is detrimental for a controllable CNT deposition, and hence must be suppressed. Furthermore, as the field frequency decreases, ion-charges within the local double layer also experience a Coulomb force, causing ions to move within the solution with respect to the direction of the electric field. This motion leads to a flow known as DC electro-osmosis (DCEO) of the liquid and should also be reduced for an ideal CNT-DEP deposition. Therefore, in order to achieve a well-aligned and highly dense CNT assembly, a complete comprehension of the fluid electrohydrodynamics involving electrical, thermal and fluidic fields is required.

\subsubsection{Potential field}

The distribution of the electromagnetic field in a liquid is derivable by solving Maxwell's equations. For a microsystem, it is reliable to neglect the magnetic effect on the flow of liquid because the energy stored in the magnetic field, $W_{\mathrm{M}}$, is much smaller than that of the electrical energy, $W_{\mathrm{E}}[35]$, as

$$
\frac{W_{\mathrm{M}}}{W_{\mathrm{E}}}=\frac{(1 / 2) \mu|\vec{H}|^{2}}{(1 / 2) \varepsilon|\vec{E}|^{2}} \ll 1
$$

Within a liquid, the conduction and displacement currents give rise to the magnetic field. If the conduction current dominates, the magnetic field intensity $|\vec{H}| \sim \sigma|\vec{E}| L$, with $L$ standing for the characteristic length of the system. Thus, for a conductivity $\sigma<0.1 \mathrm{~S} / \mathrm{m}$ and $L<1 \mathrm{~mm}$

$$
\frac{W_{\mathrm{M}}}{W_{\mathrm{E}}} \sim \frac{\mu \sigma^{2} L^{2}}{\varepsilon}<10^{-5}
$$

If the displacement current dominates, $|\vec{H}| \sim \omega \varepsilon|\vec{E}| L$. For a frequency $f=\omega / 2 \pi<10 \mathrm{MHz}$ and $L<1 \mathrm{~mm}$,

$$
\frac{W_{\mathrm{M}}}{W_{\mathrm{E}}} \sim \mu \varepsilon \omega^{2} L^{2}<10^{-5}
$$

Therefore, for a linear and isotropic medium with a displacement field of $\varepsilon \vec{E}$, the magnetic field is negligible, and Maxwell's equations approach the quasi-electrostatic limit $[35,67]$, where

$$
\begin{aligned}
\nabla \times \vec{E} & =0 \\
\nabla \cdot(\varepsilon \vec{E}) & =\rho_{\mathrm{q}}
\end{aligned}
$$




$$
\nabla \cdot \vec{j}+\frac{\partial \rho_{\mathrm{q}}}{\partial t}=0
$$

here $\rho_{\mathrm{q}}$ is the volume charge density of the medium. The Eq. (32) is the continuity equation of Maxwell's equations.

In a bulk electrolyte, the relationship between the current density $\vec{j}$ and the electrical conductivity $\sigma$ is more complicated than Ohm's law, $\vec{j}=\sigma \vec{E}$, because the current is usually transported by different types of ions with different mobilities.

For a binary symmetrical electrolyte [35], such as SDS-water, the current density $\vec{j}$ is

$$
\vec{j}=e\left(n_{+}+n_{-}\right) \mu_{\mathrm{e}} \vec{E}-e D \nabla\left(n_{+}-n_{-}\right)+e\left(n_{+}-n_{-}\right) \vec{v}
$$

with $e$ denoting the absolute value of the electric charge, and $\mu_{\mathrm{e}}, D, n_{+}, n_{-}$are the mobility, diffusion coefficient and the number density of positive and negative ions, respectively. The relative difference in the number densities of different ions is

$$
\Gamma=\frac{n_{+}-n_{-}}{n_{0}}=\frac{\nabla \cdot(\varepsilon \vec{E})}{e n_{0}} \sim \frac{\varepsilon|\vec{E}|}{e n_{0} L}
$$

with $n_{0}$ standing for the unperturbed ion density. Under the conditions of $|\vec{E}| \sim 10^{5} \mathrm{~V} / \mathrm{m}, n_{0} \sim 10^{23} \mathrm{~m}^{-3}$, and $L \sim 10 \mu \mathrm{m}$, $\Gamma$ is about $10^{-4}$, which indicates that the liquid can be considered as quasi-electroneutral, meaning the third item of Eq. (33) is negligible [68]. Furthermore, comparing the diffusion current $e D \nabla\left(n_{+}-n_{-}\right)$to the drift current $e\left(n_{+}+n_{-}\right) \mu_{\mathrm{e}} \vec{E}$ of Eq. (33) yields [35]

$$
\frac{\left|e D \nabla\left(n_{+}-n_{-}\right)\right|}{e\left(n_{+}+n_{-}\right) \mu_{\mathrm{e}}|\vec{E}|} \sim \frac{D \varepsilon|\vec{E}| / L^{2}}{2 e n_{0} \mu_{\mathrm{e}}|\vec{E}|}=\left(\frac{\lambda_{\mathrm{De}}}{L}\right)^{2}
$$

where $\lambda_{\mathrm{De}}=\sqrt{\varepsilon D / 2 e n_{0} \mu_{\mathrm{e}}}$ is the Debye length, a length scale over which mobile charge carriers screen out the electric field in solutions or in conductors. Generally, $\lambda_{\mathrm{De}}$ is about the order of nanometers for water with ionic surfactants like SDS, which is much smaller than the characteristic length (typically in microns) of a DEP system (this is not true for the toluenebased case, where $\lambda_{\mathrm{De}}$ becomes larger than the DEP characteristic length as discussed below). Hence, the ratio of Eq. (35) is very small in practice, thereby diffusion current is negligible. Furthermore, when comparing the convection current $\rho_{\mathrm{q}} \vec{v}$ from the Eq. (31) with the conduction current $\sigma \vec{E}$ $\left(\sigma=2 e n_{0} \mu_{\mathrm{e}}\right)$

$$
\frac{|\nabla \cdot(\varepsilon \vec{E}) \vec{v}|}{|\sigma \vec{E}|} \sim \frac{\varepsilon / \sigma}{L / \nu} \sim 7 \times 10^{-6}
$$

for a given $\sigma \sim 10^{-3} \mathrm{~S} / \mathrm{m}, L \sim 10 \mu \mathrm{m}$ and $v \sim 100 \mu \mathrm{m} / \mathrm{s}$. The term $\varepsilon v / L \sigma$, known as the electrical Reynolds number [69], represents the ratio of the timescale of charge convection by the fluid flow over that of charge relaxation in the Ohmic conduction. Since this value is usually small, the electrical equations are decoupled from the mechanical equations, as mentioned in Ref. [35].

Based on the above discussions, it is convenient to neglect diffusion currents and convection currents and to assume that $\sigma$ and $\varepsilon$ are time-independent within DEPs. Thus, combining Eqs. (31) and (32) as

$$
\nabla[(\sigma+i \omega \varepsilon) \vec{E}]=0
$$

with the electric field described by $\vec{E}(\omega, t)=\vec{E}_{0} e^{i \omega t}$.

In practice, the gradients in permittivity and conductivity of the fluid are minimal, and thus, the electric potential distribution is described by the Laplace equation

$$
\Delta \varphi=0
$$

\subsubsection{Thermal field}

The electric field of the DEP generates electric currents due to existing ions, which inevitably heats the liquid. In order to derive the temperature distribution of the system, one must solve the equation which describes the coupling between the internal energy of media and temperature [66]

$$
\rho_{\mathrm{m}} c_{\mathrm{p}}\left(\frac{\partial T}{\partial t}+(\vec{v} \cdot \nabla) T\right)=K \nabla^{2} T+\sigma|\vec{E}|^{2}
$$

where $c_{\mathrm{p}}$ is the specific heat capacity of the media under standard pressure, and $K$ is the thermal conductivity of the fluid. In the presence of an electric field, the temperature field rapidly reaches a stationary state within a diffusion time interval $t_{\text {diff }}=\rho_{\mathrm{m}} c_{\mathrm{p}} l^{2} / K$. It is shorter than 0.1 seconds for DEP in toluene $(K=0.141 \mathrm{~W} / \mathrm{mK}$, see Table 1$)$ and in SDS-water $(K=0.6 \mathrm{~W} / \mathrm{mK})$. Under the heating of an AC field, the total temperature $T$ becomes $T_{0}+\Delta T(t)$, where $T_{0}$ is the time average temperature and $\Delta T(t)$ is the frequencydependent temperature variation. Normally $\Delta T / T \approx 1 / 2 \omega t_{\text {diff }}$, which is negligible for an electrical field with a frequency higher than $1 \mathrm{kHz}$, meaning the item $\rho_{\mathrm{m}} c_{\mathrm{p}} \partial T / \partial t$ can be neglected from the above equation [66]. Therefore, only the time average term stays.

In a microsystem, the convection of heat is smaller than heat diffusion. This comparison is usually characterized as the Péclet number, denoting the ratio between thermal energy transmitted within fluid through convection over conduction [70], and as given by

$$
\frac{\left|\rho_{\mathrm{m}} c_{\mathrm{p}}(\vec{v} \cdot \nabla) T\right|}{\left|K \nabla^{2} T\right|} \sim P e=\frac{\rho_{\mathrm{m}} c_{\mathrm{p}}|\vec{v}| L}{K} \ll 1
$$

Neglecting the heat convection term, the temperature equation (39) reduces to the Poisson's equation, with the Joule heating as the energy source

$$
K \nabla^{2} T=-\sigma|\vec{E}|^{2}
$$

\subsubsection{Fluidic field}

Based on the mass conservation principle, the velocity $\vec{v}$ of an incompressible fluid can be described using the Navier-Stokes equations [35]

$$
\begin{gathered}
\nabla \cdot \vec{v}=0 \\
\rho_{\mathrm{m}}\left(\frac{\partial \vec{v}}{\partial t}+(\vec{v} \cdot \nabla) \vec{v}\right)=-\nabla p+\eta \nabla^{2} \vec{v}+\vec{f}_{\vec{E}}+\rho_{\mathrm{m}} \vec{g}
\end{gathered}
$$

here $\rho_{\mathrm{m}}(\partial \vec{v} / \partial t+(\vec{v} \cdot \nabla) \vec{v}),-\nabla p, \eta \nabla^{2} \vec{v}, \vec{f}_{\vec{E}}, \rho_{\mathrm{m}} \vec{g}$ represent the inertial force, pressure force, viscous force, external electrical force and gravitational force imposed on the fluid, respectively.

For a microsystem with the dimension $L<100 \mu \mathrm{m}$ and a typical value of $\vec{v}<100 \mu \mathrm{m} / \mathrm{s}$, the Reynolds number is

$$
\frac{\left|\rho_{\mathrm{m}}(\vec{v} \cdot \nabla) \vec{v}\right|}{\left|\eta \nabla^{2} \vec{v}\right|} \sim \operatorname{Re}=\frac{\rho_{\mathrm{m}}|\vec{v}| L}{\eta} \leq 0.011 \text { (water), } 0.015 \text { (toluene) }
$$

indicating that the convective-inertial force is much smaller than the force caused by the fluid viscosity, and thereby negligible. Besides, the stationary state of the fluid forms within a time interval $t=\rho_{\mathrm{m}} L^{2} / \eta$ which is usually smaller 
than 0.01 seconds after the electric field is on, indicating the term $\rho_{\mathrm{m}} \partial \vec{v} / \partial t \approx 0$ for a long time observation. Thus, the timeaveraged component of fluidic motions $\vec{v}$ can be derived using

$$
0=-\nabla p+\eta \nabla^{2} \vec{v}+\vec{f}_{\vec{E}}+\rho_{\mathrm{m}} \vec{g}
$$

For an incompressible fluid, the time average term of $\vec{f}_{\vec{E}}$ is

$$
\vec{f}_{\vec{E}}=\frac{1}{2} \Re\left(\rho_{\mathrm{q}} \vec{E}^{*}-\frac{1}{2} \vec{E} \cdot \vec{E}^{*} \nabla \varepsilon\right)
$$

The charge and electric field on the right-hand side are in complex amplitudes. The first term represents the Coulomb force, and the second term indicates the dielectric force that depends on gradients of conductivity and permittivity of the fluid.

In practice, local heating of electric currents induces gradients in the permittivity and conductivity of a fluid, which in turn induces a body force on the fluid, known as the ETF as mentioned above. Taking the effect of ETF into account, the Eq. (46) expands as $[42,66,71]$

$\vec{f}_{\vec{E}}=\frac{1}{2} \mathfrak{R}\left(\left(\left(\frac{\sigma_{\mathrm{m}} \varepsilon_{\mathrm{m}}\left(S_{1}-S_{2}\right)}{\sigma_{\mathrm{m}}+i \omega \varepsilon_{\mathrm{m}}}\right)(\nabla T \cdot \vec{E})\right) \vec{E}^{*}-\frac{1}{2} \alpha \varepsilon_{\mathrm{m}}|\vec{E}|^{2} \nabla T\right)$

with $S_{1}=(1 / \varepsilon)(\partial \varepsilon / \partial T) \approx-0.004 K^{-1}$ and $S_{2}=(1 / \sigma)(\partial \sigma / \partial T) \approx$ $0.02 K^{-1}$ standing for the relative variations of fluid permittivity and conductivity induced through the temperature gradient.

Based on the Eq. (47), it is clear that the ETF is field frequency-dependent: If $\omega \gg \sigma / \varepsilon$ (about $5.6 \mathrm{MHz}$ for 0.01-wt.\%-SDS water and $9 \mathrm{~Hz}$ for $100-\mathrm{ppm}-\mathrm{H}_{2} \mathrm{O}$ toluene in this work), then the second term (the dielectric force) dominates the ETF; Otherwise, the first term (Coulomb force) does.

Gradients of temperature can also generate a gravitational body force $\vec{f}_{\mathrm{g}}$ on the fluid, known as buoyancy. When $\omega \varepsilon / \sigma \ll 1$, the buoyancy force is smaller than the electrical force as given in the Eq. (46) since

$$
\left|\frac{\vec{f}_{g}}{\vec{f}_{\vec{E}}}\right| \sim \frac{\left(\partial \rho_{\mathrm{m}} / \partial T\right)(L)^{3}|\vec{g}|}{(1 / \sigma)(\partial \sigma / \partial T) \varepsilon V^{2}}
$$

yielding a result of $\left|\vec{f}_{g} / \vec{f}_{\vec{E}}\right|$ smaller than $10^{-3}$ for all media conditions used in this work, e.g. water and toluene with a characteristic length $L<50 \mu \mathrm{m}$ and an applied voltage $V=10 \mathrm{~V}$. If $L$ is further reduced, for instance down to micronscales and below, the influence of the buoyancy becomes negligible.

AC currents can generate divergent electric fields in the planar electrode arrays of DEP chips, which leads to electric field components tangential to the EDLs at the surface of electrodes (as shown in Fig. 2(d)). Such tangential fields exert non-zero time-averaged forces on the ions within the diffuse double layers, causing them to move along EDLs, which in turn results in a drag flow on the fluids. The induced flow, known as the ACEO flow, starts from zero at the slip-plane (a hypothetical boundary plane separating a stagnant liquid layer at the interface from the bulk liquid [72]) and gradually increase to a maximum of $v_{\mathrm{ACEO}}$ [73]. Generally, the timeaverage ACEO slip velocity can be derived through the generalization of Smoluchowski formula [52]

$$
v_{\mathrm{ACEO}}=\Lambda \frac{\varepsilon_{\mathrm{m}} V^{2}}{8 \eta x} \frac{\Gamma^{2}}{\left(1+\Gamma^{2}\right)^{2}}
$$

with a dimensionless frequency, $\Gamma$ expressed as

$$
\Gamma=\frac{\omega C_{\mathrm{DL}} \pi x}{2 \sigma_{\mathrm{m}}}
$$

$x$ denotes the distance in the horizontal direction from the center of the electrode gap. The $C_{\mathrm{DL}}$ represents the capacitance of the electrical double layer which is composed of an immobile charged Stern layer and a Poisson-Boltzmann-distribution charged diffuse layer formed at the interface between the electrolyte and electrode (as shown in Fig. 2(d)). The factor $\Lambda$ indicates the ratio of the Stern-layer capacitance $C_{\mathrm{S}}$ over the sum capacitance of the $C_{\mathrm{S}}$ and diffuse layer capacitance $C_{\mathrm{D}}$. It dominates the field of potential distribution within the EDLs and will be discussed in Section 3.

The $v_{\text {ACEO }}$ profile depends strongly on the electrical-field frequency. It tends to be zero at both low and high-frequency limits, but reaches a maximum when $\Gamma=1$. For a high field frequency, both drift and diffusion velocities of the surface charge are small, because the EDL cannot relax and rebuild sufficiently within the short time of field oscillation. Whereas at a low frequency, the applied potential $V_{\text {Applied }}$, especially in a medium with high ion-concentration, drops mainly across the double layer which results in a minimal electric potential $V_{\text {DEP }}$ across the solution.

The maximum DCEO velocity above the double layer can be determined using the Helmholtz-Smoluchowski equation $[73,74]$

$$
v_{\text {DCEO }}=-\frac{\varepsilon_{\mathrm{m}} \zeta}{\eta} E_{x}
$$

where $\zeta$ is the zeta-potential at the slip-plane, denoting the potential difference between the micelle forming surfactant solution and the dielectric layer of the substrate $[73,75] . E_{x}$ denotes the electric field tangential to the solid surface of the substrate. Unlike the ACEO, the driven potential of the DCEO across the dielectric-layer/electrolyte interface becomes negligible at high frequency, leading to an insignificant slip velocity.

\section{Experimental considerations}

\subsection{Circuit of dielectrophoresis}

In the above sections, we have assessed the physics describing the DEP-induced motion of a CNT and the competing factors. Generally, a simulation of the electric field distribution within a liquid can be obtained by numerically solving the Laplace equation of eq. (38). Such a simulation is essential since the field-magnitude distribution and frequency dominate the deposition and alignment of the CNT. A detail which complicates the electric-field simulation is the interface between the solid surface and liquid. Such an interface requires extending the simulation space for the Laplace equation beyond the liquid region using $\nabla(\varepsilon \nabla \varphi)=0$, with $\varepsilon$ the permittivity of the materials involved.

A highly conducting material, such as a metal, can be modelled as either a region with infinitely high permittivity or as surfaces with a fixed potential (Dirichlet boundary condition) [76]. However, modelling an ionic conductor under external bias is more difficult because of the charge accumulation at the liquid-metal interface, which gives rise to the formation of the EDL (as shown in Figure 2(d)) showing up as a complex impedance in the simulation. Generally, the characteristic screening length in a liquid is the Debye length $\lambda_{\mathrm{De}}=\sqrt{\varepsilon D / \sigma} \approx \lambda_{\mathrm{DL}}$, as mentioned in Section 2.2.1. It is several orders of magnitude larger than that in metal because of the low conductivity $\sigma$ and low diffusivity $D$ of ions compared to those of free electrons. 
The impedance of the EDL as an ideal capacitor is

$$
Z_{\mathrm{DL}}=\frac{1}{i \omega C_{\mathrm{DL}}}
$$

or as a constant phase element, which is more appropriate for an imperfect dielectric [52]

$$
Z_{\mathrm{DL}}=\frac{A}{C_{\mathrm{DL}}(i \omega)^{\xi}}
$$

Typically, the equivalent circuit for a liquid excluding the double-layer would be a capacitor and a resistor in parallel

$$
Z_{\mathrm{L}}=\frac{R}{1+i \omega R C}
$$

In case that $\lambda_{\mathrm{DL}}$ is much smaller than the gap $L$ between DEP electrodes, the impedance of the system then adds up to $Z=Z_{\mathrm{DL}}+Z_{\mathrm{L}}$, as shown in Fig. 3(a).

The voltage $\varphi$ that drops across the double layer is

$$
\varphi\left(\omega, Z_{\mathrm{DL}}, Z_{\mathrm{L}}\right)=\frac{Z_{\mathrm{DL}}}{Z_{\mathrm{DL}}+Z_{\mathrm{L}}} \varphi_{0}
$$

When $\lambda_{\mathrm{DL}} \ll L$, the electrical field distribution in the liquid under an external bias of $\varphi_{0}$ can be derived by solving $\varepsilon \cdot \Delta \varphi=0$ with the electrode potential set to $\varphi_{0}-\varphi$ : The EDL presents as a $2 \mathrm{D}$ layer which weakens the internal electric field.

In the other case, when $\lambda_{D L}$ is much larger than $L$, the potential drop over the distance $L$ within the double-layer is $\varphi_{0}$, and the total impedance of the system is $Z \approx Z_{L}$ as shown in Fig. 3(b). Consequently, the field distribution in the liquid is given by $\varepsilon \cdot \Delta \varphi=0$ by setting the electrode potential to $\varphi_{0}$ : the double-layer does not influence the internal electric field or the field distribution.

In the case of $\lambda_{\mathrm{DL}} \ll L$, the impedance of the EDL depends on the field frequency. Since the impedance of the circuit, $Z=Z_{\mathrm{DL}}+Z_{\mathrm{L}}$, is then complex. One must consider the generation of an electric field in a liquid for low-, mediumand high-field frequency ranges. Here two critical frequencies $\omega_{C 1}$ and $\omega_{C 2}$ (with $\omega_{C 1}<\omega_{C 2}$ ) are defined to account for two transitions in the impedance spectrum (as introduced in Section 3.3) separating these ranges.

For $0<\omega<\omega_{C 1}$, the double layer dominates the impedance of the system $Z=Z_{\mathrm{DL}}$. The external voltage drops predominantly across the EDL, leaving the liquid quasi-field free. Thus, in this low-frequency range, the double-layer capacitance dominates the total impedance, resulting in $|Z| \propto \omega^{-1}$.

For $\omega_{C 1}<\omega<\omega_{C 2}$, the impedance is dominated by the resistive part of $Z_{\mathrm{L}}$. Hence $Z$ becomes frequency independent within this medium-frequency range, and the external voltage drops mainly across the liquid. (a)

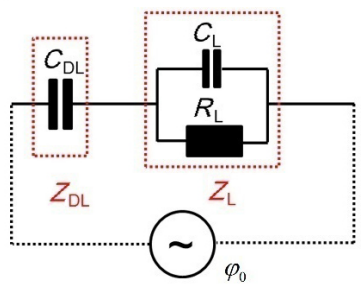

(b)

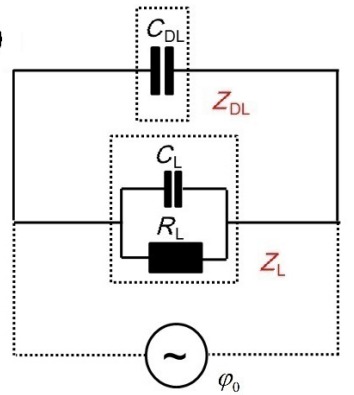

Figure 3 Equivalent circuit diagram of an electric double layer formed at the interface between DEP electrodes and liquid for (a) $\lambda_{\mathrm{DL}} \ll L$ and (b) $\lambda_{\mathrm{DL}} \gg L$
For $\omega>\omega_{C 2}$, the impedance is dominated by the capacitive component of $Z_{L}$ and again $|Z| \propto \omega^{-1}$. As in the mediumfrequency range, the external potential drops across the liquid.

The equivalent-circuit diagram of a water-based DEP system with $\lambda_{D L} \ll L$ is shown in Fig. 4(a). The magnitude and the phase of the corresponding impedance spectrum, together with the potential difference across the liquid with and without the EDL, are simulated using the commercial Multisim Analog Devices Edition 10.0 (Figs. 4(b)-4(d)) with the experimental data shown in Section 3.2.

Hence whether the applied potential $\varphi_{0}$ drops primarily across the liquid or across the EDL depends on the frequency $\omega$ and $\lambda_{\mathrm{DL}}$ in relation to the system length $L$. This dependence is schematically displayed in Table 2 .

The equivalent circuit diagram of a toluene-based DEP system with $\lambda_{\mathrm{DL}} \gg L$ and the corresponding simulations are shown in Fig. 5. In this case, all frequencies will be suitable for the maximum internal field $\left(V_{\mathrm{L}}\right)$ generation, which is opposite to the $\lambda_{\mathrm{DL}} \ll L$ case, where $\omega>\omega_{C 1}$ is required.

\subsection{Electrical double layer}

From the above discussion, it is evident that estimating the EDL thickness is very important. The screening length $\lambda_{\mathrm{De}} \approx \lambda_{\mathrm{DL}}$ and the electrode distance $L$ determine the suitable frequency range for maximizing $\mathrm{V}_{\mathrm{L}}$, thereby $V_{\mathrm{DEP}}$.

The formation of a Helmholtz EDL stems from the competition between electrostatic interactions and Brownian motion of ions as described by the Gouy and Chapman et. al. [52]. A typical representation of EDLs goes back to the Stern case, where an inner layer (called the compact layer) is in contact

Table 2 Comparisons of bias $\varphi$ across a liquid to the applied bias $\varphi_{0}$ with respect to $\lambda_{\mathrm{DL}}$ and $L$

\begin{tabular}{cccc}
\hline$\lambda_{\mathrm{DL}}$ vs. $\boldsymbol{L}$ & $\boldsymbol{\omega}<\boldsymbol{\omega}_{\mathrm{C} 1}$ & $\boldsymbol{\omega}_{\mathrm{C} 1}<\boldsymbol{\omega}<\boldsymbol{\omega}_{C 2}$ & $\boldsymbol{\omega}>\boldsymbol{\omega}_{\mathrm{C} 2}$ \\
\hline$\lambda_{\mathrm{DL}} \ll \boldsymbol{L}$ & $\varphi<\varphi_{0}$ & $\varphi \approx \varphi_{0}$ & $\varphi \approx \varphi_{0}$ \\
$\lambda_{\mathrm{DL}} \approx \boldsymbol{L}$ & $\varphi \approx \varphi_{0}$ & $\varphi \approx \varphi_{0}$ & $\varphi \approx \varphi_{0}$ \\
$\lambda_{\mathrm{DL}} \gg \boldsymbol{L}$ & $\varphi \approx \varphi_{0}$ & $\varphi \approx \varphi_{0}$ & $\varphi \approx \varphi_{0}$ \\
\hline
\end{tabular}

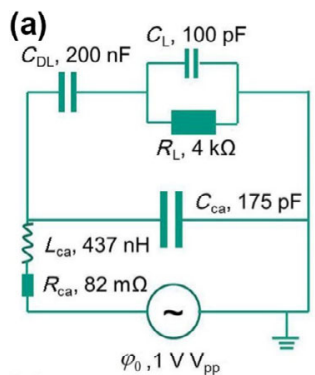

(c)
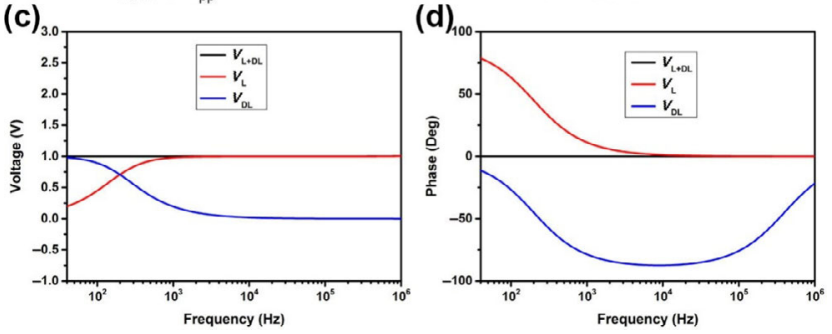

Figure 4 (a) Equivalent circuit diagram of a DEP experiment with $\lambda_{\mathrm{DL}} \ll L$, the double-layer capacitance $C_{\mathrm{DL}}$, the liquid layer capacitance $C_{\mathrm{L}}$, the liquid layer resistance $R_{\mathrm{L}}$, and the capacitance of the cables $C_{\text {ca. }}$. (b) Simulated impedance spectrum of the circuit. (c) The potential drop $V_{\mathrm{L}}$ across the liquid, $V_{\mathrm{DL}}$ across the double-layer, $V_{\mathrm{L}+\mathrm{DL}}$ across the liquid and the double layer with their corresponding phase spectra are given in (d). Parameters are taken from the analysis of corresponding measured impedance spectra described in Section 3.2. 


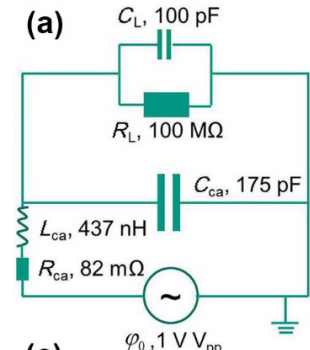

(c)

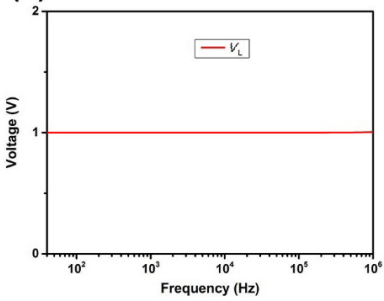

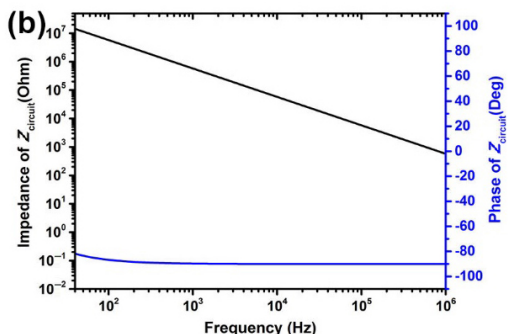

(d)

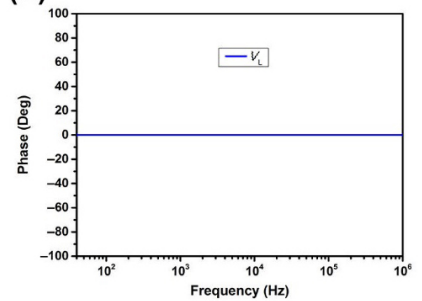

Figure 5 (a) Equivalent circuit diagram of a DEP experiment with $\lambda_{\mathrm{DL}} \gg L$. (b) Simulated impedance spectrum of the circuit. (c) The potential drop $V_{\mathrm{L}}$ across the liquid and its corresponding phase spectra (d). Parameters are taken from the analysis of corresponding impedance spectra described in Section 3.2.

with electrodes. The ions absorb on the electrode surfaces due to high electrostatic interactions. The diffuse double layer, in which the potential drops exponentially with respect to the distance, starts outside the compact layer. The potential at the interface, known as slip plane [72] formed between the compact and the diffuse layer as shown in Fig. 2(d), is called zeta potential $\zeta$ as mentioned in Section 2.2.3.

The potential distribution in the diffusive layer is derivable by solving with the non-linear Poisson-Boltzmann (PB) equation [77]

$$
\nabla \cdot(-\varepsilon \nabla \varphi)=\Sigma_{\mathrm{i}} z_{\mathrm{i}} e c_{\mathrm{i}}^{\infty} \exp \left(-z_{\mathrm{i}} e \varphi / k_{\mathrm{B}} T\right)
$$

with the bulk concentration $c_{\mathrm{i}}^{\infty}$ and charge number $z_{\mathrm{i}}$ of all ions. For a planar surface in which the thickness of EDL is negligible compared to the dimensions of its encompassing particle, meaning the thin EDL limit for a particle, the $\mathrm{PB}$ equation turns into the Gouy-Chapman (GC) equation which has an analytical solution for a semi-infinite, symmetrical binary condition of $z=z^{+}=z^{-}$, written as [77]

$$
\varphi(x)=\frac{2 k_{\mathrm{B}} T}{z e} \ln \left[\frac{1+\tanh \left(\frac{z e \varphi_{0}}{4 k_{\mathrm{B}} T}\right) \exp (-\kappa x)}{1-\tanh \left(\frac{z e \varphi_{0}}{4 k_{\mathrm{B}} T}\right) \exp (-\kappa x)}\right]
$$

with the parameter $\kappa$ equivalent to the inverse value of the Debye length

$$
\kappa^{-1}=\lambda_{\mathrm{De}}=\sqrt{\frac{\varepsilon k_{\mathrm{B}} T}{2 \mathrm{z}^{2} e^{2} c^{\infty}}}
$$

In the case of spherical geometry, meaning the thick limit of EDLs, the Debye-Hückel (DH) equation is a good approximation for the PB equation, which yields a corresponding analytical solution given by [77]

$$
\varphi(z)=\zeta \exp \left(-z / \kappa^{-1}\right)
$$

Based on the above discussion, it is apparent that the potential $\varphi$ decreases exponentially with respect to the distance from the polarized electrode. However, the DH theory becomes inappropriate if the electrical potential energy of ions is larger than the thermal energy, as in that case, one must consider the strong steric effect within EDLs.

Figure 6(a) demonstrates the excellent agreement between the $\mathrm{PB}$ equation and the analytical Eq. (57) for a case with $\varphi>k_{\mathrm{B}} T / z e$ at the room temperature. In contrast, the Debye length from the $\mathrm{DH}$ theory slightly overestimates the doublelayer thickness. However, the PB equation and Eq. (57) also have limited applicability at large surface potentials $(\varphi \gg$ $k_{\mathrm{B}} T / z e$ ) because they neglect that ions have a finite size. Therefore, the ion concentration at the electrodes can reach unrealistic values at large voltages, as shown in Figs. 6(b) and $6(\mathrm{c})$.

To account for the steric effect of ions, a modified PoissonBoltzmann (MPB) equation, which includes the influence of finite ion sizes within the Stern layer, has been proposed by Kilic et al. [77] and is given by

$\nabla \cdot(-\varepsilon \nabla \varphi)=\sum_{\mathrm{i}} z_{\mathrm{i}} e \frac{c_{\mathrm{i}}^{\infty} \exp \left(-z_{\mathrm{i}} e \varphi / k_{\mathrm{B}} T\right)}{1+2 v_{\text {ion }} \sinh ^{2}\left(z_{\mathrm{i}} e \varphi / 2 k_{\mathrm{B}} T\right)} \exp \left(z_{\mathrm{i}} e \varphi / k_{\mathrm{B}} T\right)$,

$\varphi(x=0)=\zeta, \varphi(x \rightarrow \infty)=0, \frac{\mathrm{d} \varphi}{\mathrm{d} x}(x \rightarrow \infty)=0$

Here $v_{\text {ion }}$ denotes the packing parameter $v_{\text {ion }}=2 \chi^{3} c^{\infty}$, and it can be derived by taking the effective ions size $\chi$ into account. Notably, the way to characterize $\chi$ is to consider it as a cutoff for the unphysical divergences of the PB theory. This parameter includes not only the solvation shell $\left(\approx 6.6 \AA\right.$ for $\mathrm{Cl}^{-}$and $\mathrm{OH}^{-}$ in aqueous $[52,78]$ ) but also the permittivity change induced by the electric field (up to a factor of 10 for water, as discussed below) [79], making $\chi$ larger than the measurable diameter of the ion.

For a binary symmetric z:z electrolyte, the MPB Eq. (60) becomes

$$
\nabla \cdot(-\varepsilon \nabla \varphi)=-z e c^{\infty} \frac{2 \sinh \left(z_{\mathrm{i}} e \varphi / k_{\mathrm{B}} T\right)}{1+2 v_{\text {ion }} \sinh ^{2}\left(z_{\mathrm{i}} e \varphi / 2 k_{\mathrm{B}} T\right)}
$$

Figures 6(b) and 6(c) demonstrate the limitations of the PB, GC and DH in comparison to the MPB. It is perceivable that over-packing of ions at the electrode surface occurs when raising the bias from 0.1 to $1 \mathrm{~V}$. However, the obtained characteristic thickness (thickness of Stern layer) of absorbed
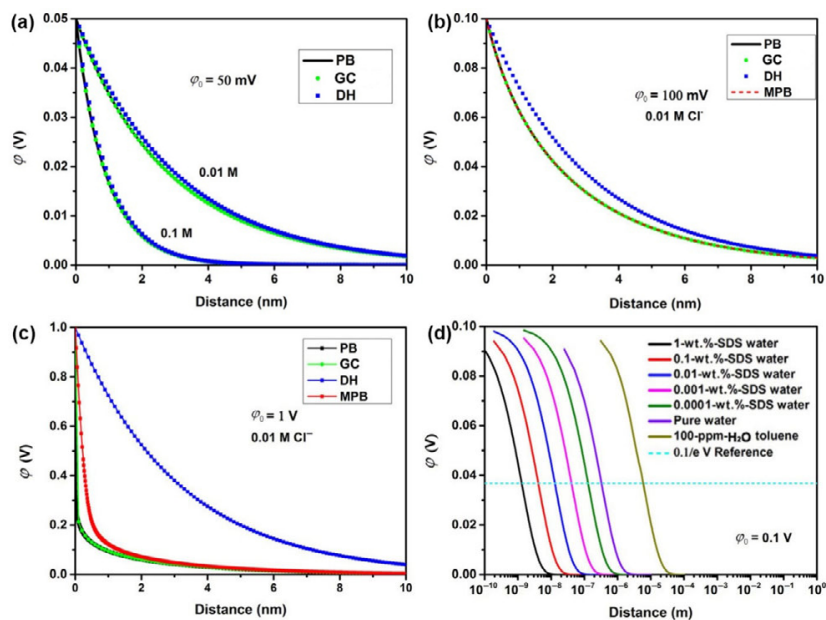

Figure 6 Comparisons of electric potential distributions calculated using the PB, GC, DH and MPB models under different (a) ion (Chlorine ion $\mathrm{Cl}^{-}$, with " $\mathrm{M}$ " standing for mole) concentrations and different bias: (a) $50 \mathrm{mV}$, (b) $100 \mathrm{mV}$ and (c) $1 \mathrm{~V}$. (d) Electric potential distribution derived with MPB model for different ion-concentration media: 1-wt.\%-SDS water, 0.1-wt.\%-SDS water, 0.01-wt.\%-SDS water, 0.001-wt.\%-SDS water, $0.0001-w t . \%-S D S$ water, pure water and $100-\mathrm{ppm}-\mathrm{H}_{2} \mathrm{O}$ toluene. The light-blue dash line denotes the electric potential at $\lambda_{\mathrm{De}}$. 
ions in the $\mathrm{PB}$ model is smaller than the one reasonably evaluated in the MPB model. Therefore, under a high bias, the MPB is appropriate to derive the potential distribution of bias across the EDLs.

From the above discussions, one can determine the double-layer thickness $\lambda_{\mathrm{DL}}$ for solutions with different ion concentrations. Figure $6(\mathrm{~d})$ shows the potential profiles starting at $\varphi_{0}=0.1 \mathrm{~V}$ across the EDLs formed in neutral water, in an aqueous surfactant solution with 1 wt. $\%$ SDS $\left(c^{\infty}=0.034 \mathrm{~mol} / \mathrm{L}\right)$, in diluted solutions (dilution factors $10,10^{2}, 10^{3}$ and $10^{4}$ ), as well as in commercial toluene with a typical impurity concentration of $100-\mathrm{ppm} \mathrm{H}_{2} \mathrm{O}$. The simulations show that the EDL thickness is on the order of $1 \mathrm{~nm}$ for solutions of 1 -wt.\%-SDS water, and about $1 \mu \mathrm{m}$ for pure water, while it is up to $10 \mu \mathrm{m}$ for toluene (here $\sigma=10^{-11} \mathrm{~S} / \mathrm{m}$ is in use). Practically, a stable aqueous dispersion of individual CNTs requires a surfactant concentration on the order of 1-wt.\% SDS, and for the dielectrophoretic deposition of CNTs, these dispersions are often used either undilutedly or diluted by a factor of up to 100 . Hence, the double-layer thickness in aqueous CNT dispersions for DEP is below $100 \mathrm{~nm}$. Since the typical electrode distance in this work is $1 \mu \mathrm{m}$, this means that the EDL thickness $\lambda_{\mathrm{DL}} \ll L$. Therefore $\varphi_{0}$ drops mainly across the double-layer but not across the liquid for $\omega<\omega_{C 1}$.

$\omega_{C 1}$ can be derived using the expression for the cutoff frequency of an RC circuit as

$$
\omega_{C 1}=\left(\frac{R_{L} C_{\mathrm{DL}}}{2}\right)^{-1}=\left(\frac{\varepsilon}{\sigma} \frac{L}{2 \lambda_{\mathrm{DL}}}\right)^{-1}
$$

with $C_{\mathrm{DL}}=\varepsilon A / \lambda_{\mathrm{DL}}$ and $R_{\mathrm{L}}=L / \sigma A, A$ is the effective crosssection area of parallel electrode pairs. $\sigma$ stands for the conductivity of the solution. $\varepsilon=81 \varepsilon_{0}$ is the permittivity of water with $\varepsilon_{0}$ the vacuum permittivity [60], see Table 1 .

An overview of $\lambda_{\mathrm{DL}}, \sigma$ and $\omega_{\mathrm{C} 1}$ for pure water and water with various concentrated SDS is given in Table 3. The calculations show that for a CNT-DEP based on aqueous dispersion with an electrode distance of $L \approx 1 \mu \mathrm{m}$, one should apply AC signals at $\omega>0.1 \mathrm{MHz}$ to ensure that the external voltage drops mainly across the liquid instead of only across the EDLs. Moreover, since electrochemical reactions, including the dissociation of water, are suppressed at AC frequencies, high peak-to-peak voltages are accessible.

In a toluene-based CNT dispersion, the situation is different. The reported value for the conductivity of toluene ranges from $\sigma=10^{-11}$ to $10^{-10} \mathrm{~S} / \mathrm{m}$ [80]. This conductivity originates from the charged impurities such as dissociated water in toluene. The solubility limit of water in toluene is $330 \mathrm{ppm}$, and even commercially available anhydrous toluene contains a significant water content $<100 \mathrm{ppm}$, which corresponds to a water concentration of $10^{-3} \mathrm{~mol} / \mathrm{L}$. However, data on the ion concentration of Toluene is absent in the literature. Therefore, one must derive the critical frequency through impedance spectroscopy measurements. Novikov et al. [80] measured a conductivity of $\sigma=10^{-10} \mathrm{~S} / \mathrm{m}$ and a critical frequency $\omega_{\mathrm{C}} \approx 1 \mathrm{~Hz}$ for toluene with parallel electrodes at a distance of

Table 3 The experimentally derived $\lambda_{\mathrm{DL}}, \sigma, \omega_{\mathrm{Cl}}$ with respect to different ion-concentration media

\begin{tabular}{cccccc}
\hline Parameters & $\begin{array}{c}\text { 1-wt.\% SDS } \\
\text { water }\end{array}$ & $\begin{array}{c}0.1-\text { wt.\% } \\
\text { SDS water }\end{array}$ & $\begin{array}{c}0.01 \text {-wt.\% } \\
\text { SDS water }\end{array}$ & Water & Toluene \\
\hline$\lambda_{\mathrm{DL}}(\boldsymbol{m})$ & $3.2 \times 10^{-9}$ & $6.0 \times 10^{-9}$ & $19.8 \times 10^{-9}$ & $3.6 \times 10^{-7}$ & $13.5 \times 10^{-6}$ \\
$\boldsymbol{\sigma}(\mathbf{S} / \mathbf{m})$ & $2.3 \times 10^{-1}$ & $2.9 \times 10^{-2}$ & $4.0 \times 10^{-3}$ & $5.5 \times 10^{-6}$ & $1 \times 10^{-10}$ \\
$\boldsymbol{\omega}_{C 1}(\mathbf{H z}) /$ & $2.1 \times 10^{6}$ & $5.0 \times 10^{5}$ & $2.3 \times 10^{5}$ & $5.5 \times 10^{3}$ & 0.038 \\
$\boldsymbol{L}(\boldsymbol{\mu m})$ & & & & & \\
\hline
\end{tabular}

$L \approx 100 \mu \mathrm{m}$. The thickness of the double-layer can be estimated using Eq. (62). Taking $\varepsilon=2.38 \varepsilon_{0}, \lambda_{\mathrm{DL}}=1.35 \cdot 10^{-5} \mathrm{~m}$ (as shown in Table 3 ) was obtained for Toluene. This calculation shows that $\lambda_{\mathrm{DL}} \gg L$ in toluene for electrodes with a gap size of $\sim 1 \mu \mathrm{m}$. Therefore, $\varphi_{0}$ drops mainly across the liquid and becomes independent of the field frequency.

Even though we have estimated the EDL thickness, which can be much larger than the electrode distance, strictly speaking, the non-linear PB and MPB models are improper for electrical potential analysis under these situations, because the deformation of EDLs induced by the convection and polarization of electrolytes are absent in these models [81]. Additionally, in the vicinity of the electrode surface, generally within the range of few nanometers, the approximation of electro-neutral liquids based on Eq. (34) becomes no longer appropriate due to charge separations induced by the local high electric field. In order to describe the behaviour of such a thick diffuse double layer, the Nernst-Planck equations for all the ions, combined with Poisson's equation, known as the Poisson-Nernst-Planck (PNP) equations, should be employed [82].

$$
\begin{gathered}
\frac{\partial c_{\mathrm{i}}}{\partial t}=\nabla \cdot\left(D_{\mathrm{i}} \nabla c_{\mathrm{i}}+\mu_{\mathrm{i}} z_{\mathrm{i}} e c_{\mathrm{i}} \nabla \varphi\right) \\
\nabla \cdot(-\varepsilon \nabla \varphi)=\sum_{\mathrm{i}} z_{\mathrm{i}} e c_{\mathrm{i}}
\end{gathered}
$$

where $\mu_{\mathrm{i}}$ is the mobility of the species $i$, and $D_{\mathrm{i}}=\mu_{\mathrm{i}} / k T$ stands for Einstein's relation. Figure 7 demonstrates the corresponding electric potential distribution of EDLs using the PNP model with concerning the upper limitation of ion-concentration given by $v_{\text {ion }}=2 \chi^{3} c^{\infty}$. The results for toluene vary somewhat as compared to Fig. 6(d), but still $\lambda_{\mathrm{DL}} \gg L$.

Regarding the Stern layer, in which immobile ions strongly adsorb to the electrode surface and implying that no free charge exists, mathematically the electric potential within this layer is also accessible based on

$$
\nabla \cdot(-\varepsilon \nabla \varphi)=0
$$

However, in practice, the permittivity of polar electrolytes is not constant when the electric field becomes very large. Because the orientation of electrolyte molecules becomes highly ordered due to the saturation of polarization under such a high electric field, resulting in the suppression of continuous orientation even with the further increase of the field amplitude. This phenomenon leads to the decrease of the medium permittivity. As Booth stated [79], there are two regions involved in the divergence of the liquid permittivity with respect to an artificial critical electric field intensity of $10^{7} \mathrm{~V} / \mathrm{m}$. Above this intensity, the permittivity of the medium is given by

$$
\varepsilon_{x}(|\vec{E}|)=n^{2}+\left(\varepsilon_{x}(0)-n^{2}\right) \frac{3}{\beta|\vec{E}|}\left[\operatorname{coth}(\beta|\vec{E}|)-\frac{1}{\beta|\vec{E}|}\right]
$$
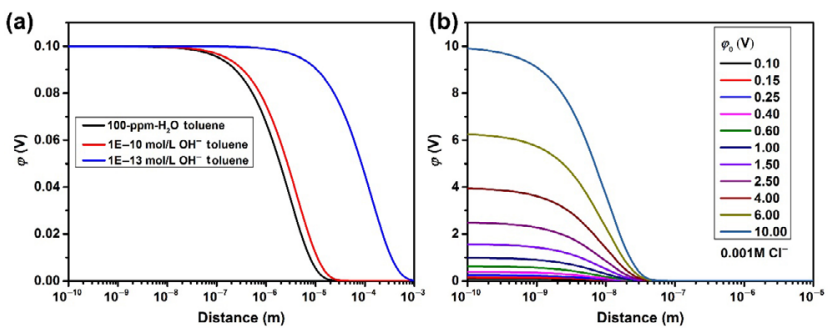

Figure 7 EDL profiles based on the Poisson-Nernst-Planck equation for different (a) ion-concentrations (100-ppm- $\mathrm{H}_{2} \mathrm{O}$ toluene, $10^{-10} \mathrm{~mol}(\mathrm{M}) / \mathrm{L}$ $\mathrm{OH}^{-}$toluene and $10^{-13} \mathrm{M} / \mathrm{L} \mathrm{OH}^{-}$toluene) and (b) surface potential (with $0.001 \mathrm{M} / \mathrm{L} \mathrm{Cl}^{-}$). 
with $\beta=5 b / 2 k T\left(n^{2}+2\right)$. While for $\left.|\vec{E}|<10^{7} \mathrm{~V} / \mathrm{m}, \varepsilon_{x}|\vec{E}|\right)$ is approximately equivalent to the bulk permittivity under zero electric field $\varepsilon_{x}(0)$, which is equivalent to the bulk fluid permittivity $\varepsilon_{\mathrm{m}}$. The parameter $n$ denotes the refractive index of the liquid [83], and $b=1.85 \times 10^{-18}$ (CGSE units, for toluene $\left.b \approx 0.36 \times 10^{-18}[84]\right)$ stands for the dipole moment of the solvent.

Figure 8 shows the calculated permittivity distributions for two kinds of electrolytes, 0.01 -wt.\%-SDS water and 100-ppm$\mathrm{H}_{2} \mathrm{O}$ toluene. When the applied potential is above $0.1 \mathrm{~V}$, the permittivity of 0.01 -wt.\%-SDS water decreases dramatically within the Stern layer (from $81 \varepsilon_{0}$ to below $30 \varepsilon_{0}$ ). In contrast, the permittivity for $100-\mathrm{ppm}-\mathrm{H}_{2} \mathrm{O}$ toluene is almost unchanged (around $2.38 \varepsilon_{0}$ ) before the potential rises to $0.5 \mathrm{~V}$. Therefore, in order to precisely derive the impedance of the EDLs, $Z_{\mathrm{DL}}=1 / i \omega C_{\mathrm{DL}}$, one must consider the calibration of the permittivity change within the Stern layer for large-bias based CNT DEPs.

The double-layer capacitance $C_{\mathrm{DL}}$ can be treated as a serial connection of the Stern layer capacitance $C_{\mathrm{S}}$ and the diffuse layer capacitance $C_{\mathrm{D}}$ and is given by

$$
\frac{1}{C_{\mathrm{DL}}}=\frac{1}{C_{\mathrm{S}}}+\frac{1}{C_{\mathrm{D}}}, C_{\mathrm{S}}=\varepsilon_{\mathrm{S}} \frac{A}{d_{\mathrm{s}}}, C_{\mathrm{D}}=\varepsilon_{\mathrm{D}} \frac{A}{d_{\mathrm{D}}}, d_{\mathrm{S}}=\chi / 2, d_{\mathrm{D}}=\lambda_{\mathrm{De}}
$$

The effective cross-section area of the parallel electrode in this work is $A=2.0 \times 10^{-5} \mathrm{~m}^{2}$ and is derived from impedance spectroscopy measurements, as discussed in the next section. Because of the capacitive impedance distribution of EDLs, the weight ratio of the electrical potential drop across the diffuse layers compared to the total potential of EDLs is $\Lambda=C_{\mathrm{S}} /\left(C_{\mathrm{D}}+C_{\mathrm{C}}\right)$, as described in Section 2.2.3. While for the Stern layer, it is $1-\Lambda$, which is $\sim 0.07$ for 0.01 -wt.\%-SDS water, and $\sim 3.5 \times 10^{-5}$ for $100-\mathrm{ppm}-\mathrm{H}_{2} \mathrm{O}$ toluene based on parameters presented in Table 1 under a bias of $0.5 \mathrm{~V}$. Considering the electrical-field-induced permittivity change, $1-\Lambda$ increases up to 0.14 for the water solution, while staying almost unchanged for the toluene case as the bias rises to $1.0 \mathrm{~V}$, because the dipole moment of molecular toluene is much smaller than that of water.

At low frequency, the capacitive impedance of the liquid bulk is much larger than the Ohmic resistance. Thus, the conductivity $\sigma_{m}$ dominates the impedance of the bulk liquid. Under that situation, the potential drop across the EDLs becomes
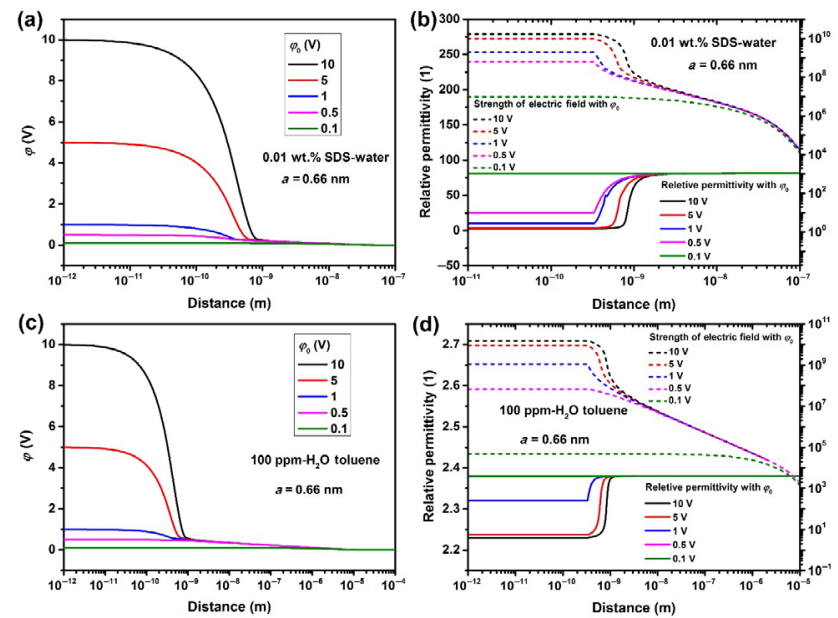

Figure 8 The calculated ((a) and (c)) electric potential, ((b) and (d)) permittivity and electric field strength distributions of 0.01-wt.\%-SDS water and $100-\mathrm{ppm}-\mathrm{H}_{2} \mathrm{O}$ toluene with taking the Stern layer into account.
$\Delta \varphi_{\mathrm{DL}}=\left(V_{\mathrm{DEP}} / 2\right) /\left(1+i \omega L C_{\mathrm{DL}} / 2 \sigma_{\mathrm{m}}\right)$ with $L$ denoting the characteristic length of the DEP circuit.

Overall, within this section, the take-home message at this point is that EDLs can significantly modify the electric field and that the applied electrical bias cannot be used entirely for the SDS-water or toluene-based CNT-DEPs. The ratio of the potential loss over the total bias is accessible through the characterization of EDL dielectrics and geometries using the MPB or PNP models. However, in order to derive to what extent the applied bias is practical for DEP, the capacitance value of the EDL is required, which can be obtained from impedance spectroscopy measurements of typical DEP circuits.

\subsection{Impedance spectroscopy}

In order to make use of impedance spectroscopy, it is crucial to understand all the impedances of the electrical elements involved in the DEP [73]. We have discussed a way to characterize the double-layer thickness and the critical frequencies for a liquid relevant to the CNT-DEP: water dispersions with ionic surfactant SDS and toluene dispersions with non-ionic polymer surfactant. For electrodes with micro-scale gaps in toluene, the applied potential always drops mainly across the bulk liquid, whereas for water, a critical frequency $\omega_{C 1}$ exists. When a low-frequency $\left(\omega<\omega_{C 1}\right)$ field is applied in water-based DEP, the potential mainly drops across the double layer, while at a high frequency $\left(\omega>\omega_{C 1}\right)$, it falls across the liquid. Experimentally, such a critical frequency is traceable in the impedance spectrum.

Figure 9 shows how the impedance spectrum of a DEP circuit varies with respect to the employed solvents. For pure water, the transition frequency occurs at $\omega / 2 \pi<1 \times 10^{4} \mathrm{~Hz}$. This transition frequency depends on the solvent conductance and rises with increasing ionic surfactant concentration, which agrees with the above discussions about the cutoff frequency $\omega_{\mathrm{C} 1}$ of the EDL (Table 3).

The experimental results are fitted based on circuit models (Fig. 4(a) and 5(a)) with taking the EDL as a constant phase element (Eq. (53)). The corresponding EDL capacitance $C_{\mathrm{DL}}$ and the exponent index $\xi$ of the high ion-concentration media have been derived using a bulk liquid capacitance $C_{\mathrm{L}}$ of $2.019 \mathrm{pF}$ (as illustrated in Table 4). Figure 9 shows an excellent agreement between the models and the experimental data. Three regimes are distinguishable in the impedance spectra for all electrolytes, except for toluene. For toluene, the impedance spectrum is entirely coincident with that of the bare circuit where no liquid bridges the electrode gap. As a result, the corresponding fitting parameters become inaccessible. This phenomenon indicates that the capacitance of the EDL for toluene is much smaller than the parasitic capacitance, verifying that the applied DEP bias mainly drops across the liquid because $\lambda_{\mathrm{DL}} \gg L$.
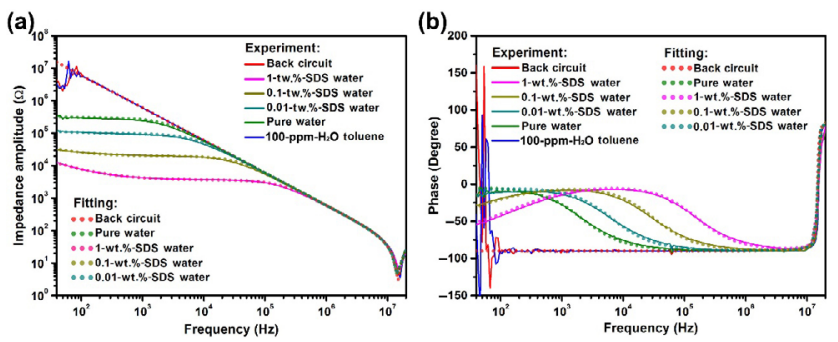

Figure 9 Experimental observations and fits of the (a) impedance amplitude and (b) phase for DEP circuits exposed to various media (1-wt.\%-SDS water, 0.1-wt.\%-SDS water, 0.01-wt.\%-SDS water, pure water and $100-\mathrm{ppm}-\mathrm{H}_{2} \mathrm{O}$ toluene). The driven bias is $0.5 \mathrm{~V}$. Back circuit stands for no liquid case. 
Table $4 R_{\mathrm{L}}, C_{\mathrm{L}}, C_{\mathrm{DL}}$ and $\xi$ of a typical DEP circuit with $L_{\mathrm{ca}}=437 \mathrm{nH}, R_{\mathrm{ca}}=3 \Omega$ and $C_{\mathrm{ca}}=263 \mathrm{pF}\left(\varphi_{0}=0.5 \mathrm{~V}\right)$ for different solvents

\begin{tabular}{cccccccc}
\hline Medium & $R_{\mathrm{L}}(\Omega)$ & $C_{\mathrm{L}}(\mathrm{pF})$ & $C_{\mathrm{DL}}(\mu \mathrm{F})$ & $\xi(1)$ & $\lambda_{\mathrm{DL}}{ }^{(\mathrm{a})}(\mathrm{nm})$ & $\lambda_{\text {De }}+\lambda_{\mathrm{S}}(\mathrm{nm})$ & $\lambda_{\mathrm{De}}+\frac{\varepsilon_{\mathrm{m}} \lambda_{\mathrm{S}}}{\varepsilon_{\mathrm{S}}}(\mathrm{nm})$ \\
\hline 1-wt.\%-SDS-Water & 3,556 & 2.019 & 2.396 & 0.6738 & 2.992 & 2.01 & 3.00 \\
0.1-wt.\%-SDS-Water & 19,290 & 2.019 & 1.201 & 0.6845 & 5.968 & 5.63 & 6.62 \\
0.01-wt.\%-SDS-Water & 88,580 & 2.019 & 0.400 & 0.6857 & 17.921 & 17.13 & 18.12 \\
\hline
\end{tabular}

${ }^{a} \lambda_{\mathrm{DL}}$ is half of the measured EDL thickness due to two symmetric EDLs formed on each interface of electrode pairs in experiments.

For a quantitative comparison, $\lambda_{\mathrm{DL}}$ for different SDS aqueous solution concentrations have been experimentally obtained and displayed in Table 4. The EDL thickness of SDS-water increases with respect to the decrease in surfactant concentration, which varies from $3.0 \mathrm{~nm}$ for $1-\mathrm{wt} . \%-S D S$ water to $17.9 \mathrm{~nm}$ for $0.01-w t . \%$-SDS water. Besides, considering the relative permittivity change induced by the high local electric field within the Stern layers, it is evident that the value of $\lambda_{\mathrm{De}}+\lambda_{\mathrm{S}} \varepsilon_{\mathrm{m}} / \varepsilon_{\mathrm{S}}$ gives a better evaluation of the $\lambda_{\mathrm{DL}}$ than $\lambda_{\mathrm{De}}+\lambda_{\mathrm{S}}$. This kind of difference becomes more pronounced when a more highly polarizable solvent is used in the DEP.

Furthermore, taking a closer look at the derived exponent index $\xi$, we note that this parameter rises slightly from 0.6738 for 1 -wt.\%-SDS water up to 0.6851 for 0.01 -wt.\%-SDS water, indicating that the dielectric EDL becomes more lossless when the ion-concentration decreases. Following this change, the entire EDL thickness rises from $0.006 \mu \mathrm{m}$ up to $0.036 \mu \mathrm{m}$, which means that the capacitance $C_{\mathrm{L}}$ of aqueous bulk can no longer be considered constant when the electrolyte becomes heavily diluted. This change leads to uncertainties in measuring the capacitance of the liquid and thereby the thickness of the EDLs.

\section{Implications for carbon nanotube dielec- trophoresis}

Based on the previous discussions, we will now simulate, as a practical example, the deposition of carbon nanotubes dispersed both in ionic surfactant solutions and in toluene for single and multiple contact geometries and verify the results with experimental observations.

\subsection{CNT suspensions}

The experiments employ two types of CNT dispersions, 0.01-wt.\%-SDS water and toluene with about 100 ppm $\mathrm{H}_{2} \mathrm{O}$. The preparation process of these dispersions via the sizeexclusion-chromatography (SEC) methods is described in the Refs. $[34,60]$. Absorption spectra of the dispersions are shown in Fig. 10. Both the mono-chiral and multi-chiral s-SWCNT dispersions are comparable regarding overall nanotube concentrations.

We have prepared dispersions with an average CNT length of $\sim 1 \mu \mathrm{m}$ to match the electrode gaps size, and a concentration
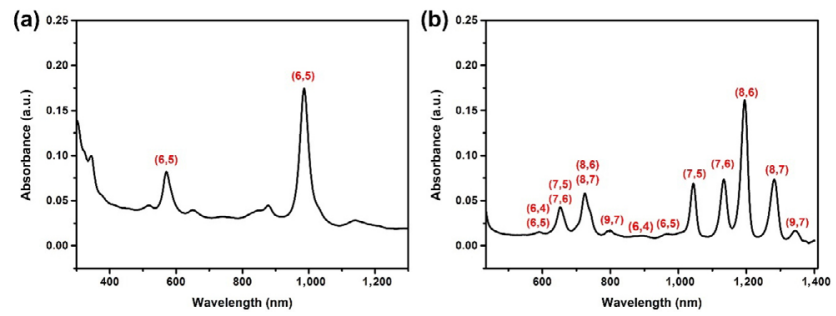

Figure 10 Optical absorption spectra of CNT dispersions based on (a) 1-wt.\%-SDS water and (b) 100-ppm- $\mathrm{H}_{2} \mathrm{O}$ toluene. The peaks are assigned to the chiral index of SWCNTs. of about $1 \mathrm{CNT}$ per $\mu \mathrm{m}^{3}$ to ensure the deposition of CNTs one-by-one. Based on results from the experiments and simulations in Ref. [60], we use conductivity and permittivity values of CNTs, as displayed in Table 1 , to describe the polarizability of the nanotubes: $\sigma=4 \times 10^{-3} \mathrm{~S} / \mathrm{m}, \varepsilon=81 \varepsilon_{0}$ for 0.01-wt.\%-SDS water (diluted with distilled water (VWR CA1.16754.5000)), and $\sigma=2 \times 10^{-10} \mathrm{~S} / \mathrm{m}, \varepsilon=2.38 \varepsilon_{0}$ for the $100-$ ppm $-\mathrm{H}_{2} \mathrm{O}$ toluene (commercial toluene (Alfa Aesar, CASnumber 108-88-3) with 100 ppm-water content).

\subsection{Device geometry}

The equations discussed in Section 2.2 for solving the electric field, temperature field, and fluid-velocity distributions of a DEP system are coupled with each other and can be solved sequentially for specific DEP cases. In order to conduct the numerical simulations, finite element method (FEM) simulations were performed using a commercial software package (COMSOL MULTIPHYSICS 5.0). A simplified schematic geometry for CNT-DEP without loss of generality is given in Fig. 11(a) (side view) and 11(b) (top view). Upon setting up appropriate boundary conditions (as mentioned below) and structured meshes (minimum mesh size of $0.1 \mathrm{~nm}$ ), the electric potential, temperature, and fluid velocity distributions arising from the applied $V_{\text {DEP }}$ become derivable through the FEM analysis.

In the following discussion, both the field-frequency dependent translational and rotational motions of CNTs with $1 \mu \mathrm{m}$ in length and $1.3 \mathrm{~nm}$ in diameter are simulated during the DEP process. Furthermore, the efficiency and alignment performance of CNT deposition using different DEPs are discussed with respect to media (either water or toluene) and electrical bias (AC or DC, $1 \mathrm{~V}_{\text {rms }}$ or $5 \mathrm{~V}_{\text {rms }}$ ).

\subsection{Potential field}

\subsubsection{Boundary condition}

In order to simplify comparisons between the effects of different electric fields, such as AC or DC, on the CNT-DEP deposition, all biases involved are given as the root-mean-square voltage

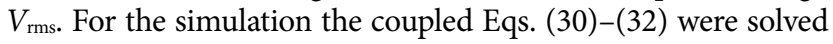
for the applied voltage (see Eq.(69)) using the insulating outer boundary conditions $\mathbf{n} \cdot \mathbf{J}=0$.

Considering the presence of EDLs between the metallic surface and the bulk electrolyte, the boundary condition for an electrode is chosen based on the charge conservation equation for the given double layer, i.e. the current flowing into the element of the double layer is equal to the increase of the stored charges. In terms of a high ion-concentration medium based CNT-DEP, the electrode gap is much larger than the EDL thickness. For instance, in the 0.01-wt.\%-SDS water-based DEP (with $\lambda_{\mathrm{DL}}$ around $17 \mathrm{~nm}$, as shown in Table 1), the EDL is theoretically equivalent to a distributed capacitor between the electrode and the bulk since the lateral currents along the double layer (either convection or conduction) is negligible in comparison to the normal current [35]. Thus, the normal current flowing into the EDL is equal to the increase of the stored charges. Besides, the thickness of the EDL is so 

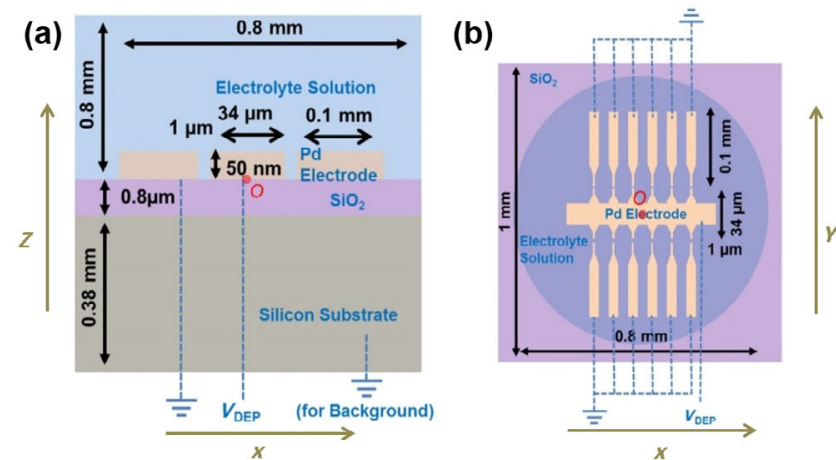

(c)

Figure 11 The side (a) and top view (b) of the DEP structure used in this work. Notably, the electrical signal is applied to the middle-common electrode. For the BG-DEP, the silicon substrate is set to the ground ("O" stands for the original point), while for the TG case, all floating electrodes are set to the ground. (c) SEM image of typical BG toluene-CNT-DEP deposition using a $50 \mathrm{~Hz}, 3.5 \mathrm{~V}_{\mathrm{rms}}$ bias based on the $90-\mu \mathrm{m}$ long, $1-\mu \mathrm{m}$ wide electrode structure (scalar bar: $1 \mu \mathrm{m}$ ).

small that it does not enter the liquid space. Therefore, the conservation of charge condition under this case is

$$
\sigma_{\mathrm{m}} \frac{\partial \varphi}{\partial n_{\perp}}=\frac{\partial}{\partial t}\left(C_{\mathrm{DL}}\left(\varphi-V_{\text {Applied }}\right)\right)
$$

where $n_{\perp}$ represents the outer normal, $C_{\mathrm{DL}}$ is the EDL capacitance per unit area and is given by $C_{\mathrm{DL}}=\varepsilon_{\mathrm{m}} /\left(\lambda_{\mathrm{D}}+\varepsilon_{\mathrm{m}} \chi\right)$ $\left.2 \varepsilon_{S}\right) . V_{\text {Applied }}$ is the electrical potential applied for the DEP. $\varphi$ denotes the electric potential just outside the double layer. With a complex amplitude, the boundary condition for $\varphi$ at the electrode surface turns to a mixed boundary condition as

$$
\varphi-\frac{\sigma}{i \omega C_{\mathrm{DL}}} \frac{\partial \varphi}{\partial n_{\perp}}=V_{\text {Applied }}
$$

For a field with frequency $\omega>\sigma / \varepsilon$, where the displacement current dominates, the potential $\varphi$ just outside the EDL becomes equal to the applied potential $V_{\text {Applied }}$. This $\varphi$ is crucial for successfully achieving CNT-DEP deposition in 0.01-wt.\%-SDS water.

However, for a DEP in 100-ppm- $\mathrm{H}_{2} \mathrm{O}$ toluene, the above concerns about the EDL boundary condition are invalid, since the thickness of the double layer is much larger than the characteristic length of DEP system ( $L=1 \mu \mathrm{m}$ in this work). In this case, a realistic description of EDL must consider the impact of the Stern layer and hence Eq. (69) has to be used to determine the electric field, thereby replacing $C_{\mathrm{S}}$ with $C_{\mathrm{DL}}$. The electrode surface potential is set through

$$
\varphi-\frac{\sigma}{i \omega C_{\mathrm{S}}} \frac{\partial \varphi}{\partial n_{\perp}}=V_{\text {Applied }}
$$

For the substrate/electrolyte interface within the electrodes gap, the boundary condition is given by the continuity of the total normal current density using

$$
\left(\sigma_{\mathrm{SiO}_{2}}+i \omega \varepsilon_{\mathrm{SiO}_{2}}\right) \frac{\partial \varphi_{\mathrm{SiO}_{2}}}{\partial n_{\perp}}=\left(\sigma_{\mathrm{m}}+i \omega \varepsilon_{\mathrm{m}}\right) \frac{\partial \varphi_{\mathrm{m}}}{\partial n_{\perp}}
$$

Two typical wiring configurations are employed in this work for CNT-DEPs, back-grounded (BG) and top-grounded (TG) as shown in Figs. 11(a) and 11(b). The former indicates that the conducting silicon wafer is set to the ground potential, which is the configuration that has been applied for wafer-scale CNT-DEP deposition [85]. Under this situation, all floating electrodes, excluding the common one in Figs. 11(a) and 11(b), are modelled at floating potentials by using the electric shielding boundary condition provided by COMSOL. The TG connection means that all floating electrodes are set to the ground potential, while the common electrode is fixed with a potential derived from the Eqs. (69) or (70).

\subsubsection{Simulation and discussion}

Figures 12 and 13 show the side-view potential distributions

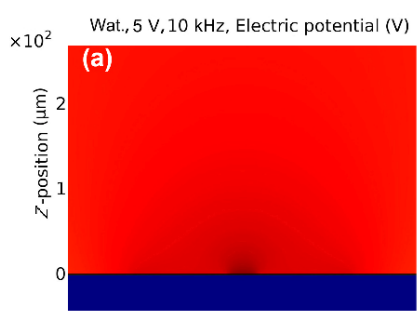

Wat, 5 V, $100 \mathrm{kHz}$, Electric potential (V)

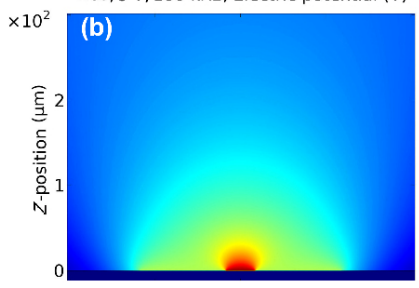

Wat., $5 \mathrm{~V}, 1 \mathrm{MHz}$, Electric potential $(\mathrm{V})$
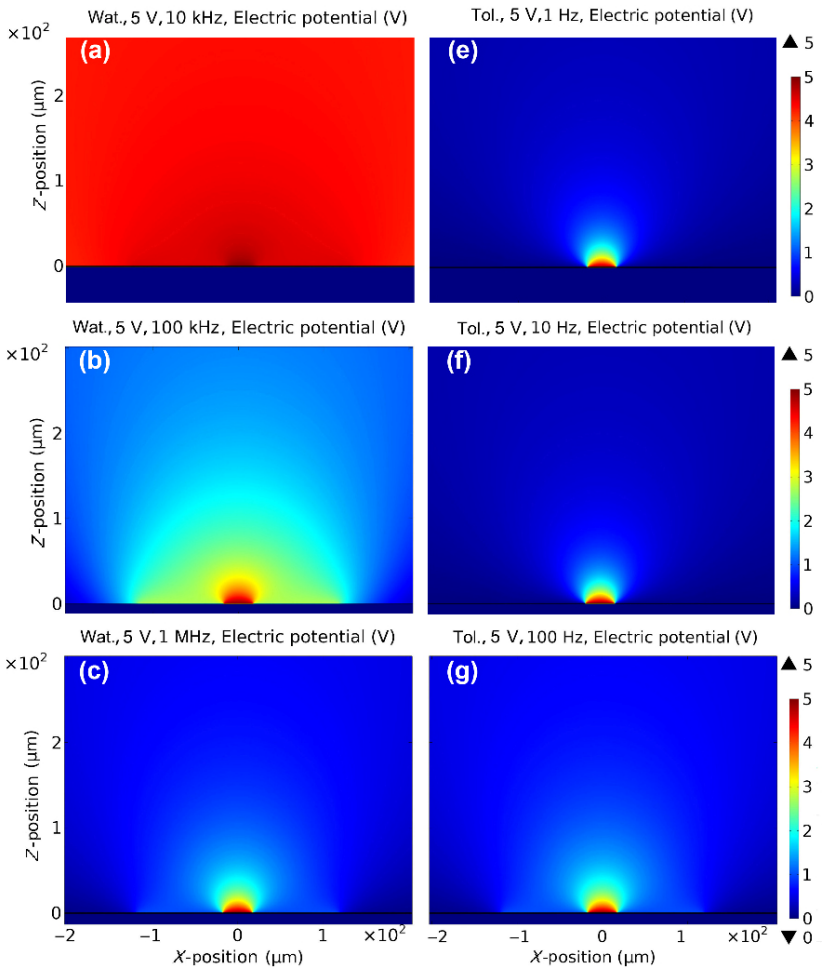

Tol., 5 V, $10 \mathrm{~Hz}$, Electric potential (V)

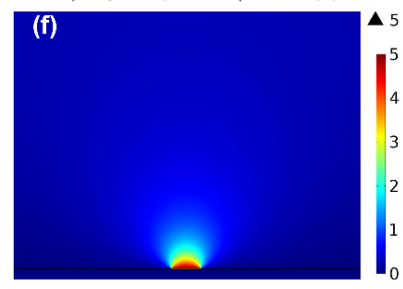

Tol., $5 \mathrm{~V}, 100 \mathrm{~Hz}$, Electric potential (V)

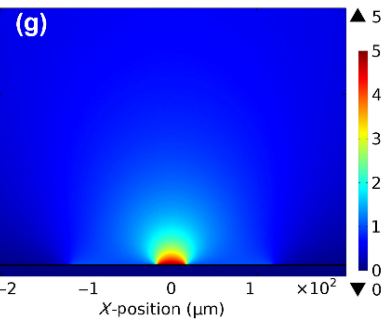

Wat, $5 \mathrm{~V}$, Electric potential $(\mathrm{V})$

Tol., 5 V, Electric potential (V)

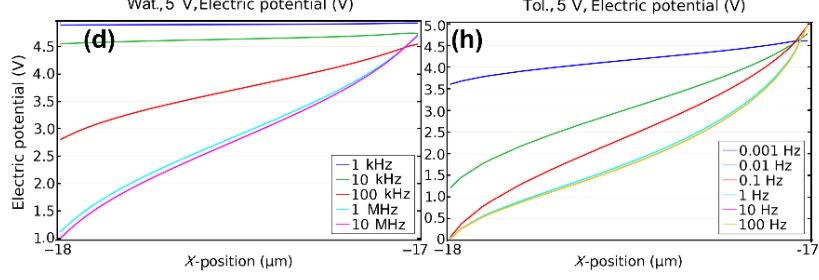

Figure 12 Back-grounded case: Characterizations of electric potential distributions for 0.01-wt.\%-SDS water (Wat.) and 100-ppm- $\mathrm{H}_{2} \mathrm{O}$ toluene (Tol.) based DEPs with respect to the field frequency. For the former, it shows the BG based potential-distribution surfaces with respect to frequencies of (a) $10 \mathrm{kHz}$, (b) $100 \mathrm{kHz}$ and (c) $1 \mathrm{MHz}$, and (d) plots of the potential drop across the electrode gap under different frequencies $(1 \mathrm{kHz}, 10 \mathrm{kHz}$, $100 \mathrm{kHz}, 1 \mathrm{MHz}$ and $10 \mathrm{MHz}$ ). For the latter, the corresponding BG based potential-distribution surfaces with respect to frequencies of (e) $1 \mathrm{~Hz}$, (f) $10 \mathrm{~Hz}$, and (g) $100 \mathrm{~Hz}$, and (h) plots of the potential drop across the electrode gap under different frequencies $(0.001 \mathrm{~Hz}, 0.01,1,10$ and $100 \mathrm{~Hz})$ are demonstrated. A bias of $5 \mathrm{~V}_{\mathrm{rms}}$ is applied for all cases. 
Wat. $5 \mathrm{~V}, 1 \mathrm{kHz}$. Electric potential (V)

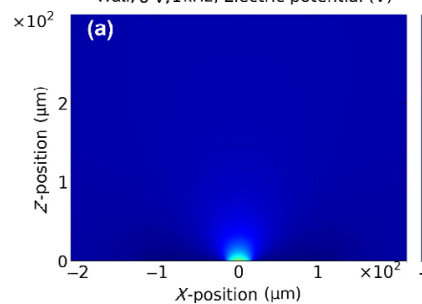

Tol, 5 V, DC, Electric potential (V)

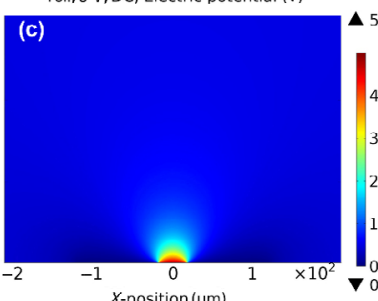

Wat., $5 \mathrm{~V}$,Electric potential $(\mathrm{V})$

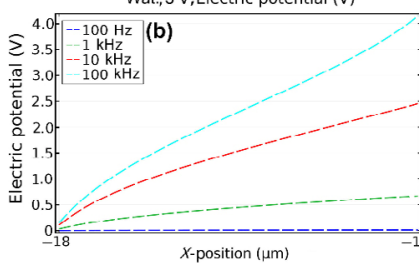

$x$-position $(u m)$

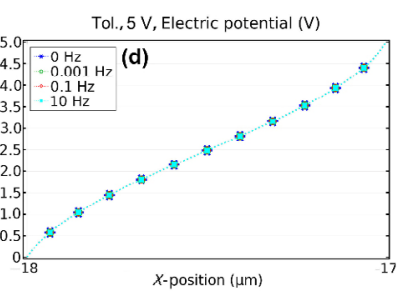

Figure 13 Top-grounded case: Characterizations of electric potential distributions for 0.01-wt.\%-SDS water (Wat.) and 100-ppm- $\mathrm{H}_{2} \mathrm{O}$ toluene (Tol.) based DEPs with respect to the field frequency. The TG based (a) potential-distribution surface under the frequency of $1 \mathrm{kHz}$ and (b) plots of the potential drop across the electrode gap with respect to frequencies of $100 \mathrm{~Hz}, 1 \mathrm{kHz}, 10 \mathrm{kHz}$ and $100 \mathrm{kHz}$ are also displayed. The TG based (c) potential-distribution surface under the DC case and (d) plots of the potential drop across the electrode gap with respect to DC and frequencies of $0.001,0.01,1$ and $10 \mathrm{~Hz}$ are also displayed. A bias of $5 \mathrm{~V}_{\mathrm{rms}}$ is applied for all cases.

of $5 \mathrm{~V} \mathrm{~V}_{\text {rms }}$ DEP both in the 0.01-wt.\%-SDS water and in the $100-$ ppm- $\mathrm{H}_{2} \mathrm{O}$ toluene at different frequencies. For the BG configuration, as shown in Fig. 12, the colour surfaces (a)-(c) demonstrate the potential distribution within the water-based DEPs as compared to the toluene case, as shown in the (e)-(g). It is evident that the potential drop mainly occurs near the electrode gap in toluene-based DEPs while it shows an apparent frequency dependence in the water case and becomes less electrode-gap-localized at low frequencies. This observation is also consistent with the frequency-dependency of the electrical potential drop across the electrode gap (location of $x:-18 \mu \mathrm{m}$ to $-17 \mu \mathrm{m}, z$ : $50 \mathrm{~nm}$ ) as shown in Figs. 12(d) and 12(h). In BG-configuration, the decreasing potential drop across the electrode gap with decreasing field frequency can be attributed to a lack of capacitive coupling between the floating electrodes and the grounded back-gate. Note that the impedance of the 800 -nm-thick $\mathrm{SiO}_{2}$ layer of the silicon wafer is in series with the impedance of the liquid between the biased common electrode and the floating electrode, effectively forming a voltage divider. which seriesly splits more potential from the $V_{\text {Applied }}$ when the frequency decreases. Furthermore, it is noteworthy that for DEPs in 100-ppm- $\mathrm{H}_{2} \mathrm{O}$ toluene, there is about $5 \mathrm{~V}_{\text {rms }}$ potential drop across the electrode gap at frequencies above $0.01 \mathrm{~Hz}$, while for the $0.01-w t . \%$-SDS water-based case, a frequency of above $10^{5} \mathrm{~Hz}$ is needed to maintain such a potential drop. This difference is due to the low conductivity of toluene as compared to water, making the impedance of toluene comparable to the impedance of the $\mathrm{SiO}_{2}$ dielectric layer even at low frequencies.

In order to confirm the feasibility of performing lowfrequency CNT-DEPs using toluene, a BG-toluene DEP with a bias of $50 \mathrm{~Hz}$ and $3.5 \mathrm{~V}_{\text {rms }}$ was performed using an extendedwidth $(90 \mu \mathrm{m})$ electrode structure, and a typical SEM observation of the deposition is shown in Fig. 11(c). Evidently, CNTs are deposited successfully under such condition. This achievement verifies the access of low-frequency CNT-DEP with a BG connection based on toluene. However, such low-frequency DEPs are inaccessible in water, because, on the one hand, the electrical potential drop mainly occurs across the EDLs instead of the electrode gap, and on the other hand, the electrolysis of metal electrode hinders the application of large bias in the water-based DEP.

As discussed in the Eqs. (15) and (16), a lower electric field frequency can result in a larger $\mathrm{CMF}$ and hence a more efficient DEP. However, the potential drop across the electrode gap declines with decreasing frequency. In order to maintain enough potential drop across the liquid at a low frequency, one approach is to change the grounding connection from the BG to the TG (used in all the following discussions). In this way, the loss of potential within the dielectric layer of the wafer is methodologically avoided. Figures 13 display the potential characterizations of CNT-DEPs based on the two kinds of media with the TG configuration. For the TG-DEP with 0.01-wt.\%-SDS water, the operating frequency can be decreased by one order of magnitude as compared to the BG-DEP case (Figs. 13(a) and $13(\mathrm{~b})$ ). However, the frequency cannot go below $10 \mathrm{kHz}$ since by then half of the potential has already been lost within the EDLs. By comparison, for DEP of $100-$ ppm- $\mathrm{H}_{2} \mathrm{O}$ Toluene (Figs. 13(c) and 13(d)), the electric field can be fully maintained for very low frequencies, even for a static DC bias. The case of low-frequency access for toluene is attributed to the thickness of EDLs being much larger than the electrode gap, making the impedance of the circuit dominated by the capacitance of the diffuse layer rather than the Stern layer or the liquid. This realization underlines the advantage of using Toluene for the CNT DEP as it allows a low-frequency bias and hence a highly-efficiency s-SWCNT deposition as discussed in the session 2.1.1.

\subsection{Thermal field}

We now discuss the influence of the thermal field on the CNT deposition in DEPs based on water and toluene.

\subsubsection{Boundary condition}

The continuity of temperature and the normal heat flux at the interface determines the boundary conditions in the temperature-field simulation. For a micro-structure, the temperature field is sensitive to the ambient environment, which can differ from one experiment to the another. As shown in Fig. 11(b), since the electrodes are only $50 \mathrm{~nm}$ thick, they can be assumed to be transparent for the flux of heat, therefore they are removable from the model [35]. Besides, the upper and lower boundaries of the model are also set to be at room temperature $(300 \mathrm{~K})$ due to the efficient heat exchange with the environment in reality. A symmetric boundary condition of $-n_{\perp} \cdot(-K \nabla T)=0$ is applied to the lateral edges, because these edges are far away from the location of power dissipation near the electrode gap [73].

\subsubsection{Simulation and discussion}

Figure 14 displays the simulated results for the steady-state temperature field from Eq. (39) on the basis of the electric field simulation. It is evident that for an AC DEP in 0.01-wt.\%-SDS water using the TG configuration, there is a significant Joule heating, thus an increase in the local temperature. The temperature gradient is much larger than for the DC DEP in 100-ppm- $\mathrm{H}_{2} \mathrm{O}$ toluene. Notably, in the water case, the temperature increases from 300 up to $301.1 \mathrm{~K}$, while it remains almost unchanged in the toluene case. Moreover, the maximum temperature gradient in water is about six orders of magnitude higher than that in toluene. As discussed in Section 2.2, such DEP-induced temperature gradients inevitably give rise to the electrothermal motion of the fluid. As shown in Fig. 14, this motion is much more pronounced in the AC DEP based on water than the DC DEP using toluene. 

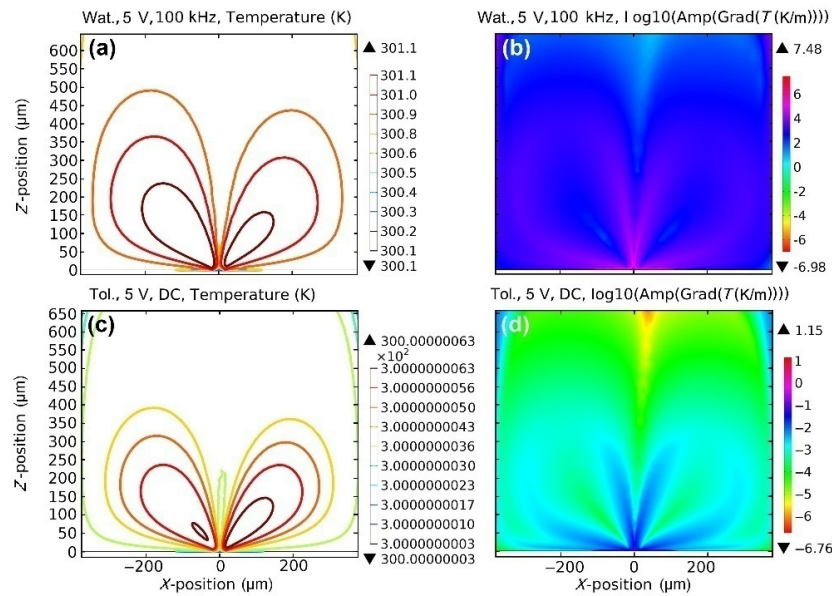

1.15

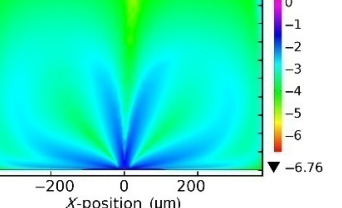

Figure 14 Side views $(y=0 \mu \mathrm{m})$ of temperature and temperature gradient distributions induced by the AC-DEP with a bias of $5 \mathrm{~V}_{\mathrm{rms}}, 100 \mathrm{kHz}$ using 0.01-wt.\%-SDS water and by the DC-DEP with a $5 \mathrm{~V}$ bias based on $100-$ ppm- $\mathrm{H}_{2} \mathrm{O}$ toluene. Both are with the TG-configuration. (a) and (c) Temperature distribution in water and toluene, respectively. (b) and (d) temperature gradient on the logarithmic scale for water and toluene, respectively.

\subsection{Fluidic field}

By setting up boundary conditions, one can calculate the velocity of the fluid based on discussions in Section 2.2.3 using Eqs. (42) and (43) on the basis the electric field and temperature distribution.

\subsubsection{Boundary condition}

No-slip conditions are imposed on all boundaries of the fluid domain: $v=0$. In particular situations, however, it is necessary to introduce a slip condition on the metallic electrodes in order to take the emergence of the ACEO into account, especially for the frequency regime when $v_{0}=v_{\mathrm{ACEO}}$. Similarly, when a DC offset is imposed between two electrodes, a slip condition on the silicon-dioxide substrate must be introduced to account for the DCEO where $v_{0}=v_{\mathrm{DCEO}}$.

In this section, again, two DEP situations are included: the AC DEP based on water and the DC DEP using toluene. For a low ion-concentration medium such as toluene, the ACEO can be neglected even under a high frequency because the thickness of EDLs is much larger than the channel length, making the $C_{\mathrm{D}}$ much smaller than that of the high ion-concentration medium. Thus, we have applied the ACEO condition for the AC DEP of surfactant-water (for instance 0.01-wt.\%-SDS water), whereas the DCEO condition for the DC DEP with toluene in the simulations.

\subsubsection{Simulation and discussion}

Figure 15 demonstrates the fluid velocity distributions present in the DEPs of 0.01-wt.\%-SDS water and of 100-ppm- $\mathrm{H}_{2} \mathrm{O}$ toluene when considering the effects of ETF, ACEO and DCEO. The steady-state fluid velocity induced in the AC DEP based on 0.01 -wt.\%-SDS water is over ten times larger than that of the DC DEP using 100-ppm- $\mathrm{H}_{2} \mathrm{O}$ toluene. This can be attributed to the pronounced ETF effect in the former case. Notably, the direction (represented by the red arrows) of the liquids just above the substrate is opposite from the left half to the right one (Fig. 15). This phenomenon is helpful to transport CNTs into the effective deposition region. Since the velocities of the fluid convection in these DEPs are overwhelming compared to the CNT diffusion (around $\sim \mu \mathrm{m} / \mathrm{s}$, caused by the Brownian motion as introduced in Section 2.1.1), it is the fluid convection

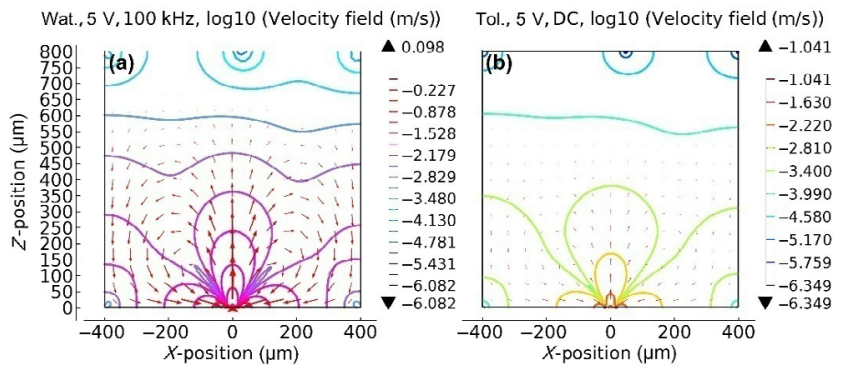

Figure 15 Side views $(y=0 \mu \mathrm{m})$ of fluid velocity induced (a) by the AC-DEP with a $5 \mathrm{~V}_{\mathrm{rms}}, 100 \mathrm{kHz}$ bias in 0.01-wt.\%-SDS water and (b) by the DC-DEP with a $5 \mathrm{~V}$ bias in $100-\mathrm{ppm}-\mathrm{H}_{2} \mathrm{O}$ toluene, both are with TG-configuration. Arrows indicate the direction of fluid flow. Note the logarithmic scale.

instead of diffusion that carries CNTs into the DEP effective region for deposition.

\subsection{Carbon nanotube dielectrophoretic deposition}

Finally, the velocity and alignment of a CNT in the DEP based on either 0.01 -wt.\%-SDS water or $100-\mathrm{ppm}-\mathrm{H}_{2} \mathrm{O}$ toluene are simulated using the TG configuration, as shown in Fig. 16. From the side-view, it is evident that the toluene DEP yields a higher deposition velocity and a higher degree of CNT alignment than the water case under the identical bias condition ( $5 \mathrm{~V} \mathrm{~V} V_{\text {rms, }}$ $100 \mathrm{kHz}$, as shown in Figs. 16(a) and 16(b); 16(i) and 16(j)). These results are expected since the detrimental properties of aqueous surfactant solutions, such as high fluid conductivity and extensive screening the CNT polarizability, lower the deposition performance of DEP. The simulations also illustrate the benefits of using the low-frequency field in the CNT DEP based on toluene, as shown in Figs. 16(e) and 16(f); 16(i) and $16(\mathrm{j}))$. For a DEP with $5 \mathrm{~V}$ DC bias, a maximum velocity of about $\sim 1 \mathrm{~m} / \mathrm{s}$ and an alignment angle (with respect to the $\mathrm{X}$-axis) of $0.01^{\circ}$ are observed, whereas, with a bias of $5 \mathrm{~V}_{\text {rms, }}$, $100 \mathrm{kHz}$, they are only $0.27 \mathrm{~m} / \mathrm{s}$ and $0.05^{\circ}$, respectively. This frequency dependence is attributed to the decrease of the CNT polarizability with respect to the increase of the MaxwellWagner relaxation field frequency, as discussed in the Ref. [60]. Similar observations are also visible in the top views of DEPs, as shown in Figs. 16(c) and 16(d), (g)-(h) and (k)-(l).

In order to further evaluate the DEP performance using these two kinds of solvents, more simulations with different bias were performed, and the results are presented in Figs. 16(c) and 16(d), 16(g) and 16(h), 16(k) and 16(l). As shown, a maximum velocity of $10^{-1.33} \mathrm{~m} / \mathrm{s} \approx 4.7 \mathrm{~cm} / \mathrm{s}$ and a highest alignment (with respect to the $X$-axis) of $85^{\circ}$ for the CNT deposition has been reached in the toluene-based DEP with $1 \mathrm{~V}$-DC bias. They become $31 \mu \mathrm{m} / \mathrm{s}$ and $63^{\circ}$ for the DEP of SDS-water using bias of $5 \mathrm{~V} V_{\text {rms }}, 100 \mathrm{kHz}$. But the two values of the latter case are comparable to $65 \mu \mathrm{m} / \mathrm{s}$ and $62^{\circ}$ derived from the $1 \mathrm{~V}-100 \mathrm{kHz}$ biased DEP in toluene. Thus, one can conclude that the toluene-based DC DEP, with an applied bias below the threshold voltage of electrolysis, is the most effective way to deposit aligned CNTs.

Aiming at quantitatively comparing the CNT deposition efficiency via DEP in SDS-water and in toluene, the corresponding velocity distributions were calculated, with the DEP induced motion subtracted from the Brownian motion, as shown in Fig. 17. The mean radius of effective regions where CNTs can be deposited effectively through the DEP with a $5 \mathrm{~V}, 100 \mathrm{kHz}$ bias in 0.01-wt.\%-SDS water is about $3 \mu \mathrm{m}$ (Fig. 17(a)). This value is only one-fourth of that for the DEP in 100-ppm- $\mathrm{H}_{2} \mathrm{O}$ toluene with a $5 \mathrm{~V}$ DC bias (Fig. 17(b)). Hence, approximately, the volume in which CNT depositions occur is about two 

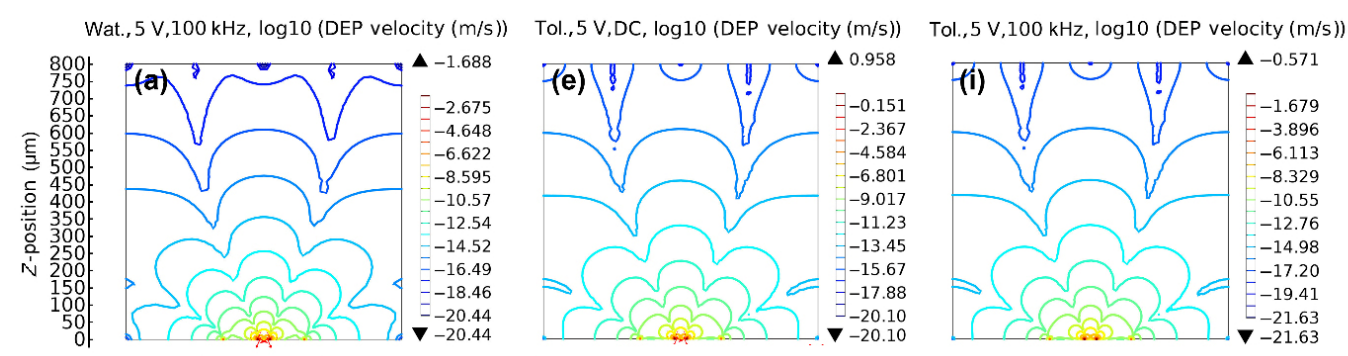

Wat., $5 \mathrm{~V}, 100 \mathrm{kHz}$, Abs(Alignment angle (Deg
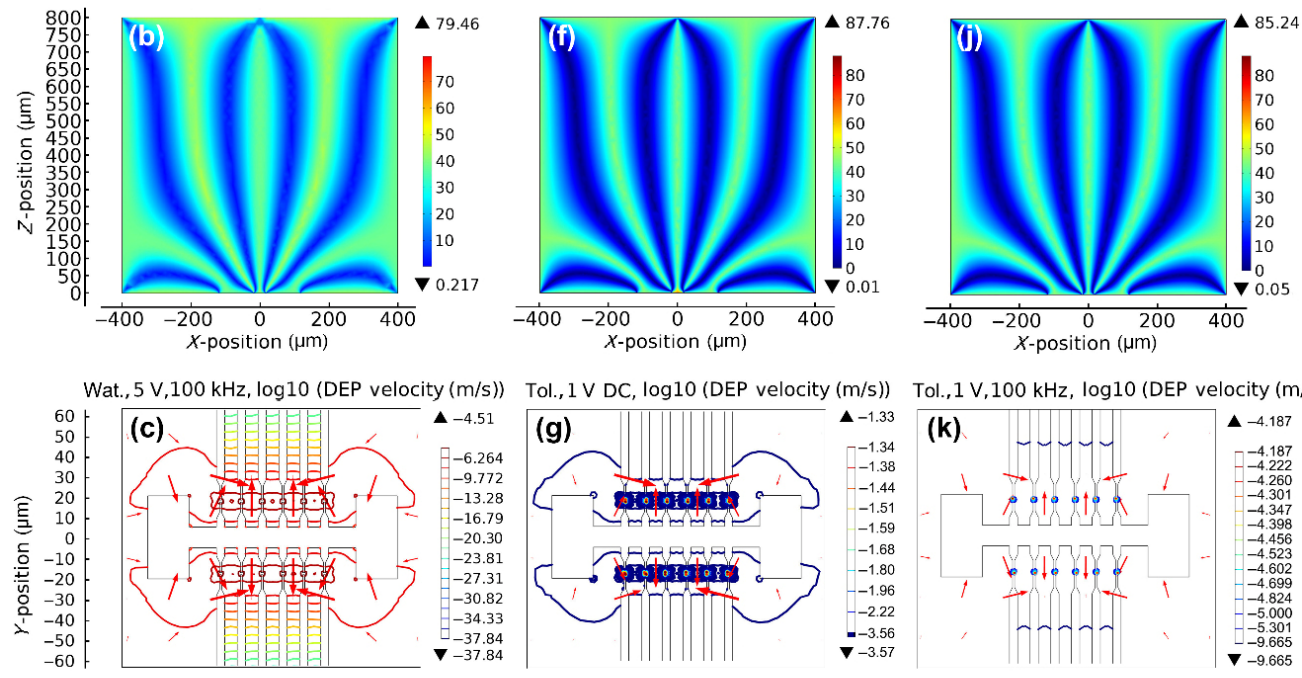

Tol., $1 \mathrm{~V}, 100 \mathrm{kHz}, \log 10$ (DEP velocity $(\mathrm{m} / \mathrm{s}))$
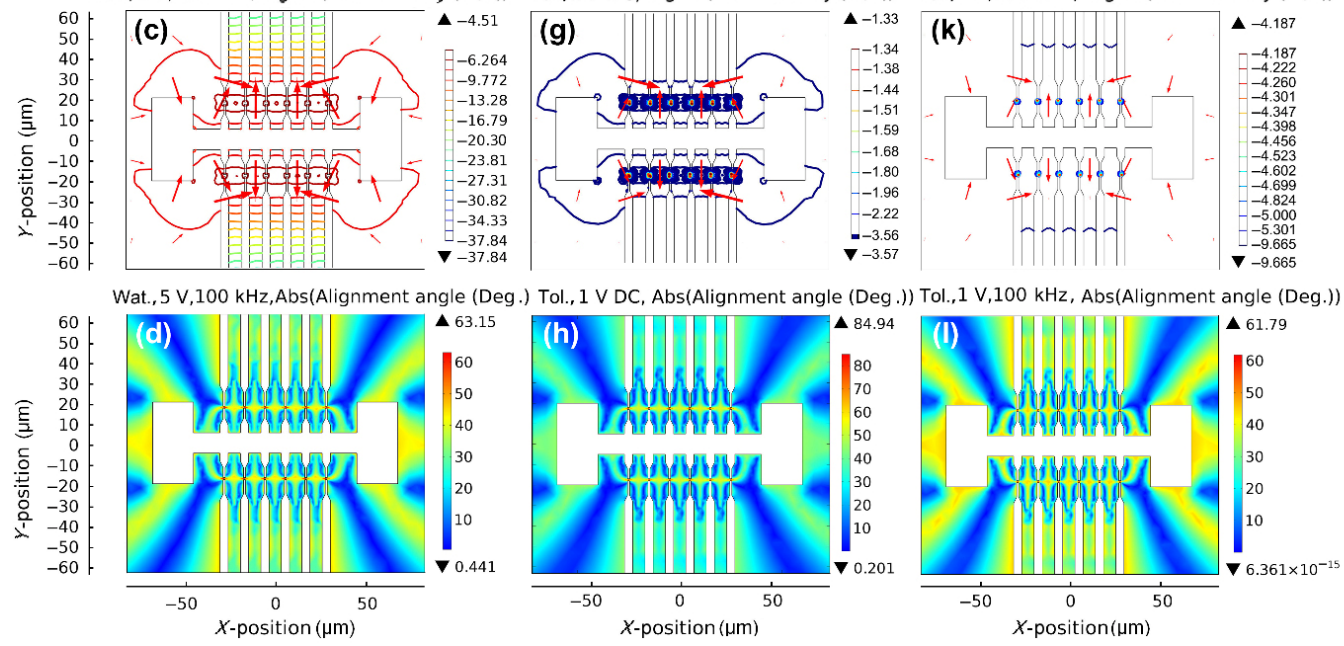

Figure 16 Simulations of the CNT velocity and alignment during DEPs in 0.01-wt.\%-SDS water and in 100-ppm- $\mathrm{H}_{2} \mathrm{O}$ toluene using TG-configuration. Side view: (a) and (b) show the logarithmic velocity contours of the CNT and its steady-state alignment on the surface (with respect to $X$-axis) in 0.01-wt.\%-SDS water with a $5 \mathrm{~V}_{\mathrm{rms}}, 100 \mathrm{kHz}$ bias. Similar is shown for the CNT dispersed in 100-ppm- $\mathrm{H}_{2} \mathrm{O}$ toluene but exposed to a $5 \mathrm{~V}$ DC bias in (e) and (f) and a $5 \mathrm{~V}_{\text {rms, }}, 100 \mathrm{kHz}$ bias in (i) and (j). The corresponding top view representation of the data is shown in (c), (d), (g), (h) and (k) and (l), respectively, with a reduced bias amplitude of $1 \mathrm{~V}$ for the $(\mathrm{g}),(\mathrm{h}),(\mathrm{k})$, and $(\mathrm{l})$.
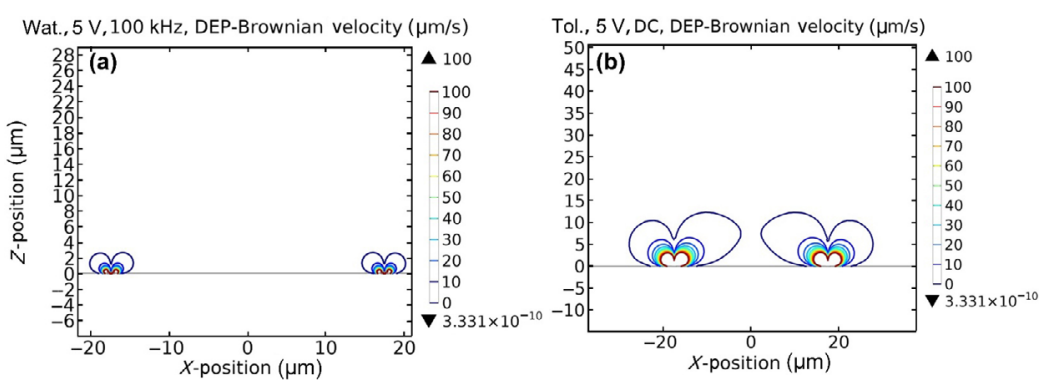

Figure 17 Side view $(y=0 \mu \mathrm{m})$ of the CNT velocity contours of TG-DEP minus the Brownian motion for (a) 0.01-w.\%-SDS-water and (b) 100-ppm- $\mathrm{H}_{2} \mathrm{O}$ toluene-based dispersions using biases of $5 \mathrm{~V}_{\mathrm{rms}}, 100 \mathrm{kHz}$ and $5 \mathrm{~V}$, DC respectively.

orders of magnitude larger for the DEP in toluene than in SDS-water.

It is noteworthy that all the above discussions are based on simulations without CNT bridging between electrodes. After the deposition of nanotubes, the electrical potential distribution between electrode gaps changes due to the electric screening of deposited CNTs, influencing the continuous assembly of CNTs [85]. Thus, further analysis is required for this situation.

Experimentally, we have verified the simulations of DEP in
0.01-wt.\%-SDS water $\left(5 V_{\text {rms }}, 100 \mathrm{kHz}\right)$ and 100-ppm- $\mathrm{H}_{2} \mathrm{O}$ toluene $(5 \mathrm{~V}, \mathrm{DC})$ with standard electrode structures (with a channel size of $1 \mu \mathrm{m} \times 1 \mu \mathrm{m}$ ). SEM images after depositions are shown in Fig. 18. As stated above, it is evident that the alignment and density of CNT deposition by DEP in 0.01-wt.\%-SDS water is worse and lower than those obtained with DEP in 100 -ppm- $\mathrm{H}_{2} \mathrm{O}$ toluene under the same or even lower bias conditions.

Finally, in order to achieve large-scale or highly dense CNT 

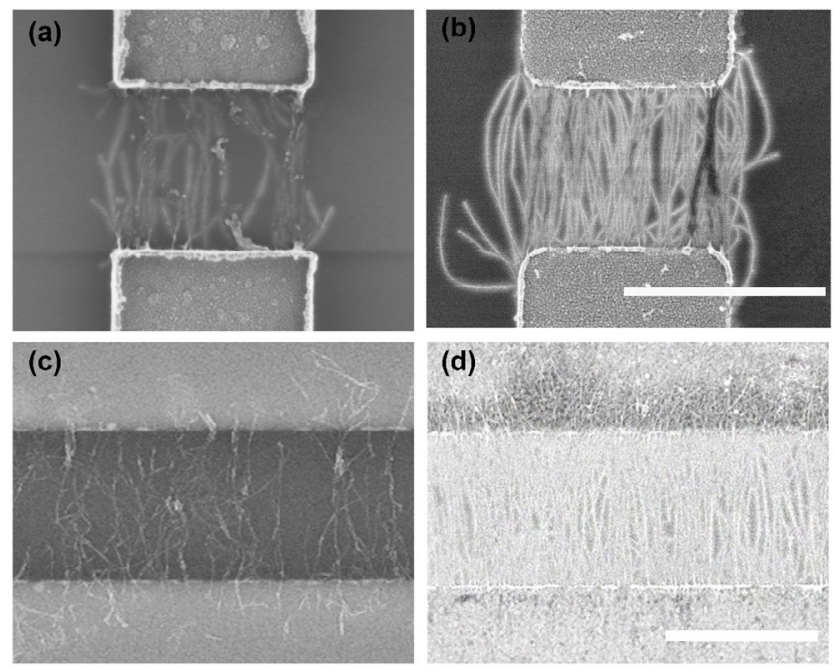

(d)

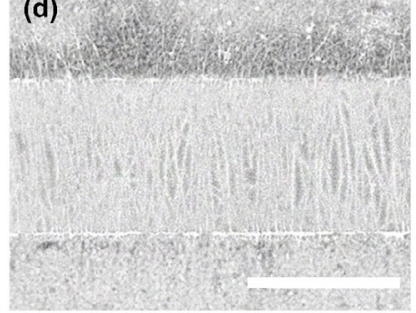

Figure 18 SEM images of CNTs deposited in narrow and wide channels by DEPs in 0.01-wt.\%-SDS water using (a) $1 \mu \mathrm{m} \times 1 \mu \mathrm{m}(5 \mathrm{~V} \mathrm{~V}$ rms, $100 \mathrm{kHz}),\left(\right.$ c) $1 \mu \mathrm{m} \times 90 \mu \mathrm{m}\left(15 \mathrm{~V}_{\mathrm{rms}}, 100 \mathrm{kHz}\right)$ structures, and in $100 \mathrm{ppm}-\mathrm{H}_{2} \mathrm{O}$ toluene with (b) $1 \mu \mathrm{m} \times 1 \mu \mathrm{m}$ (5 V, DC), (d) $1 \mu \mathrm{m} \times 90 \mu \mathrm{m}$ $(10 \mathrm{~V}, \mathrm{DC})$ structures (scalar bar: $1 \mu \mathrm{m})$. The devices are biased in the TG configuration.

deposition for optoelectrical applications, an electrode structure with a $1 \mu \mathrm{m} \times 90 \mu \mathrm{m}$ channel size is also employed for study, and the deposition results are shown in Figs. 18(c) and 18(d)). Also, in this case, the DEP in toluene $(10 \mathrm{~V}, \mathrm{DC})$ results in a CNT deposition with higher density and better alignment than that based on SDS-water $\left(15 \mathrm{~V}_{\mathrm{rms}}, 100 \mathrm{kHz}\right)$, which once again confirms the numerical simulations.

\section{Conclusions}

The principle of CNT-DEP has been discussed extensively in both theory and experiment for two solvents, water, and toluene (with 100-ppm- $\mathrm{H}_{2} \mathrm{O}$ impurity). For the toluene case, the EDL thickness turns out to be much larger than the micron-scale electrode gap. Thus, the DEP can be performed at a low frequency, even DC bias. This condition is beneficial to enhance the CNT polarizability for the DEP deposition by making use of its low-frequency CMF limit. Simulations and SEM characterizations, indeed, confirm that the performance of the DEP in toluene for s-SWCNT deposition is enhanced as compared to the DEP in water. In particular, CNT-DEP in toluene with a DC bias is shown to be the most promising approach for aligned s-SWCNT deposition. Moreover, a quantitative efficiency-difference between toluene-based DCDEP and water-based AC-DEP is demonstrated by considering the effective dielectrophoretic region for nanotube deposition.

\section{Acknowledgements}

The authors acknowledge support from the Helmholtz Research Program Science and Technology of Nanosystems (STN). B. S. Flavel acknowledges support from the Deutsche Forschungsgemeinschafts Emmy Noether Program under grant number FL 834/1-1. The COMSOL simulation code files are available online at https://tuprints.ulb.tu-darmstadt.de/id/eprint/5984.

Funding note: Open access funding enabled and organized by Projekt DEAL.

Open Access This article is licensed under a Creative Commons Attribution 4.0 International License, which permits use, sharing, adaptation, distribution and reproduction in any medium or format, as long as you give appropriate credit to the original author(s) and the source, provide a link to the Creative Commons licence, and indicate if changes were made.

The images or other third party material in this article are included in the article's Creative Commons licence, unless indicated otherwise in a credit line to the material. If material is not included in the article's Creative Commons licence and your intended use is not permitted by statutory regulation or exceeds the permitted use, you will need to obtain permission directly from the copyright holder.

To view a copy of this licence, visit http://creativecommons.org/licenses/by/4.0/.

\section{References}

[1] Dürkop, T.; Getty, S. A.; Cobas, E.; Fuhrer, M. S. Extraordinary mobility in semiconducting carbon nanotubes. Nano Lett. 2004, 4, 35-39.

[2] Cao, Q.; Han, S. J.; Tersoff, J.; Franklin, A. D.; Zhu, Y.; Zhang, Z.; Tulevski, G. S.; Tang, J. S.; Haensch, W. End-bonded contacts for carbon nanotube transistors with low, size-independent resistance. Science 2015, 350, 68-72.

[3] Kocabas, C.; Kim, H. S.; Banks, T.; Rogers, J. A.; Pesetski, A. A.; Baumgardner, J. E.; Krishnaswamy, S. V.; Zhang, H. Radio frequency analog electronics based on carbon nanotube transistors. Proc. Natl. Acad. Sci. 2008, 105, 1405-1409.

[4] Steiner, M.; Engel, M.; Lin, Y. M.; Wu, Y. Q.; Jenkins, K.; Farmer, D. B.; Humes, J. J.; Yoder, N. L.; Seo, J. W. T.; Green, A. A. et al. Highfrequency performance of scaled carbon nanotube array field-effect transistors. Appl. Phys. Lett. 2012, 101, 053123.

[5] Bachtold, A.; Hadley, P.; Nakanishi, T.; Dekker, C. Logic circuits with carbon nanotube transistors. Science 2001, 294, 1317-1320.

[6] Shulaker, M. M.; Hills, G.; Patil, N.; Wei, H.; Chen, H. Y.; Wong, H. S. P.; Mitra, S. Carbon nanotube computer. Nature 2013, 501, 526-530.

[7] Avouris, P.; Freitag, M.; Perebeinos, V. Carbon-nanotube photonics and optoelectronics. Nat. Photonics 2008, 2, 341-350.

[8] Jariwala, D.; Sangwan, V. K.; Lauhon, L. J.; Marks, T. J.; Hersam, M. C. Carbon nanomaterials for electronics, optoelectronics, photovoltaics, and sensing. Chem. Soc. Rev. 2013, 42, 2824-2860.

[9] Cao, Q.; Tersoff, J.; Farmer, D. B.; Zhu, Y.; Han, S. J. Carbon nanotube transistors scaled to a 40-nanometer footprint. Science 2017, 356, 1369-1372.

[10] Qiu, C. G.; Zhang, Z. Y.; Xiao, M. M.; Yang, Y. J.; Zhong, D. L.; Peng, L. M. Scaling carbon nanotube complementary transistors to 5-nm gate lengths. Science 2017, 355, 271-276.

[11] Shulaker, M. M.; Hills, G.; Park, R. S.; Howe, R. T.; Saraswat, K.; Wong, H. S. P.; Mitra, S. Three-dimensional integration of nanotechnologies for computing and data storage on a single chip. Nature 2017, 547, 74-78.

[12] Cao, Y.; Brady, G. J.; Gui, H.; Rutherglen, C.; Arnold, M. S.; Zhou, C. W. Radio frequency transistors using aligned semiconducting carbon nanotubes with current-gain cutoff frequency and maximum oscillation frequency simultaneously greater than $70 \mathrm{GHz}$. ACS Nano 2016, 10, 6782-6790.

[13] Rutherglen, C.; Kane, A. A.; Marsh, P. F.; Cain, T. A.; Hassan, B. I.; AlShareef, M. R.; Zhou, C. W.; Galatsis, K. Wafer-scalable, aligned carbon nanotube transistors operating at frequencies of over $100 \mathrm{GHz}$. Nat. Electron. 2019, 2, 530-539.

[14] Kshirsagar, C.; Li, H.; Kopley, T. E.; Banerjee, K. Accurate intrinsic gate capacitance model for carbon nanotube-array based FETs considering screening effect. IEEE Electron Device Lett. 2008, 29, 1408-1411.

[15] Cao, Q.; Han, S. J.; Tulevski, G. S. Fringing-field dielectrophoretic assembly of ultrahigh-density semiconducting nanotube arrays with a self-limited pitch. Nat. Commun. 2014, 5, 5071.

[16] Sun, Y. N.; Kursun, V. N-Type carbon-nanotube MOSFET device profile optimization for very large scale integration. Trans. Electr. Electron. Mater. 2011, 12, 43-50. 
[17] Ding, L.; Tselev, A.; Wang, J. Y.; Yuan, D. N.; Chu, H. B.; McNicholas, T. P.; Li, Y.; Liu, J. Selective growth of well-aligned semiconducting single-walled carbon nanotubes. Nano Lett. 2009, 9, 800-805.

[18] Che, Y. C.; Wang, C.; Liu, J.; Liu, B. L.; Lin, X.; Parker, J.; Beasley, C.; Wong, H. S. P.; Zhou, C. W. Selective synthesis and device applications of semiconducting single-walled carbon nanotubes using isopropyl alcohol as feedstock. ACS Nano 2012, 6, 7454-7462.

[19] Hong, S. W.; Banks, T.; Rogers, J. A. Improved density in aligned arrays of single-walled carbon nanotubes by sequential chemical vapor deposition on quartz. Adv. Mater. 2010, 22, 1826-1830.

[20] Ding, L.; Yuan, D. N.; Liu, J. Growth of high-density parallel arrays of long single-walled carbon nanotubes on quartz substrates. $\mathrm{J}$. Am. Chem. Soc. 2008, 130, 5428-5429.

[21] Chen, Y. B.; Zhang, Y. Y.; Hu, Y.; Kang, L. X.; Zhang, S. C.; Xie, H. H.; Liu, D.; Zhao, Q. C.; Li, Q. W.; Zhang, J. State of the art of single-walled carbon nanotube synthesis on surfaces. Adv. Mater. 2014, 26, 5898-5922.

[22] Liu, W. M.; Zhang, S. C.; Qian, L.; Lin, D. W.; Zhang, J. Growth of high-density horizontal SWNT arrays using multi-cycle in-situ loading catalysts. Carbon 2020, 157, 164-168.

[23] LeMieux, M. C.; Roberts, M.; Barman, S.; Jin, Y. W.; Kim, J. M.; Bao, Z. N. Self-sorted, aligned nanotube networks for thin-film transistors. Science 2008, 321, 101-104.

[24] Wang, Y. L.; Pillai, S. K. R.; Chan-Park, M. B. High-performance partially aligned semiconductive single-walled carbon nanotube transistors achieved with a parallel technique. Small 2013, 9, 29602969.

[25] Cao, Q.; Han, S. J.; Tulevski, G. S.; Zhu, Y.; Lu, D. D.; Haensch, W. Arrays of single-walled carbon nanotubes with full surface coverage for high-performance electronics. Nat. Nanotechnol. 2013, 8, 180-186.

[26] Li, X. L.; Zhang, L.; Wang, X. R.; Shimoyama, I.; Sun, X. M.; Seo, W. K.; Dai, H. J. Langmuir-Blodgett assembly of densely aligned single-walled carbon nanotubes from bulk materials. J. Am. Chem. Soc. 2007, 129, 4890-4891.

[27] Joo, Y.; Brady, G. J.; Arnold, M. S.; Gopalan, P. Dose-controlled, floating evaporative self-assembly and alignment of semiconducting carbon nanotubes from organic solvents. Langmuir 2014, 30, 3460-3466.

[28] Liu, L. J.; Han, J.; Xu, L.; Zhou, J. S.; Zhao, C. Y.; Ding, S. J.; Shi, H. W.; Xiao, M. M.; Ding, L.; Ma, Z. et al. Aligned, high-density semiconducting carbon nanotube arrays for high-performance electronics. Science 2020, 368, 850-856.

[29] Brady, G. J.; Way, A. J.; Safron, N. S.; Evensen, H. T.; Gopalan, P.; Arnold, M. S. Quasi-ballistic carbon nanotube array transistors with current density exceeding Si and GaAs. Sci. Adv. 2016, 2, e1601240.

[30] Maune, H. T.; Han, S. P.; Barish, R. D.; Bockrath, M.; Goddard III, M. B. W. A.; Rothemund, P. W. K.; Winfree, E. Self-assembly of carbon nanotubes into two-dimensional geometries using DNA origami templates. Nat. Nanotechnol. 2010, 5, 61-66.

[31] Zhao, M. Y.; Chen, Y. H.; Wang, K. X.; Zhang, Z. X.; Streit, J. K.; Fagan, J. A.; Tang, J. S.; Zheng, M.; Yang, C. Y.; Zhu, Z. et al. DNAdirected nanofabrication of high-performance carbon nanotube fieldeffect transistors. Science 2020, 368, 878-881.

[32] Sun, W.; Shen, J.; Zhao, Z.; Arellano, N.; Rettner, C.; Tang, J. S.; Cao, T. Y.; Zhou, Z. Y.; Ta, T. A.; Streit, J. K. et al. Precise pitch-scaling of carbon nanotube arrays within three-dimensional DNA nanotrenches. Science 2020, 368, 874-877.

[33] Keren, K.; Berman, R. S.; Buchstab, E.; Sivan, U.; Braun, E. DNAtemplated carbon nanotube field-effect transistor. Science 2003, 302, 1380-1382.

[34] Hennrich, F.; Li, W. S.; Fischer, R.; Lebedkin, S.; Krupke, R.; Kappes, M. M. Length-sorted, large-diameter, polyfluorene-wrapped semiconducting single-walled carbon nanotubes for high-density, short-channel transistors. ACS Nano 2016, 10, 1888-1895.

[35] Castellanos, A.; Ramos, A.; González, A.; Green, N. G.; Morgan, H. Electrohydrodynamics and dielectrophoresis in microsystems: Scaling laws. J. Phys. D: Appl. Phys. 2003, 36, 2584-2597.

[36] Lin, Y. Modeling of dielectrophoresis in micro and nano systems. Doctoral thesis, KTH Royal Institute of Technology, Stockholm, Sweden, 2008.

[37] Pohl, H. A. Dielectrophoresis: The Behavior of Neutral Matter in Nonuniform Electric Fields; Cambridge University Press: Cambridge, 1978.
[38] Krupke, R.; Hennrich, F.; Lohneysen, H. V.; Kappes, M. M. Separation of metallic from semiconducting single-walled carbon nanotubes. Science 2003, 301, 344-347.

[39] Krupke, R.; Hennrich, F.; Weber, H. B.; Kappes, M. M.; Löhneysen, $\mathrm{H}$. V. Simultaneous deposition of metallic bundles of single-walled carbon nanotubes using Ac-dielectrophoresis. Nano Lett. 2003, 3, $1019-1023$.

[40] Pohl, H. A. The motion and precipitation of suspensoids in divergent electric fields. J. Appl. Phys. 1951, 22, 869-871.

[41] Pethig, R. Dielectric and Electronic Properties of Biological Materials; John Willey \& Sons: New York, 1979.

[42] Green, N. G.; Ramos, A.; González, A.; Castellanos, A.; Morgan, H. Electrothermally induced fluid flow on microelectrodes. J. Electrostat. 2001, 53, 71-87.

[43] Green, N. G.; Morgan, H. Dielectrophoretic separation of nanoparticles. J. Phys. D: Appl. Phys. 1997, 30, L41.

[44] Hughes, M. P. Dielectrophoretic behavior of latex nanospheres: Lowfrequency dispersion. J. Colloid Interface Sci. 2002, 250, 291-294.

[45] Krupke, R.; Hennrich, F.; Weber, H. B.; Beckmann, D.; Hampe, O.; Malik, S.; Kappes, M. M.; Löhneysen, H. V. Contacting single bundles of carbon nanotubes with alternating electric fields. Appl. Phys. A 2003, 76, 397-400.

[46] Krupke, R.; Hennrich, F.; Kappes, M. M.; Löhneysen, H. V. Surface conductance induced dielectrophoresis of semiconducting singlewalled carbon nanotubes. Nano Lett. 2004, 4, 1395-1399.

[47] Li, W. S.; Pyatkov, F.; Dehm, S.; Flavel, B. S.; Krupke, R. Deposition of semiconducting single-walled carbon nanotubes using light-assisted dielectrophoresis. Phys. Status Solidi B 2014, 251, 2475-2479.

[48] Gomulya, W.; Gao, J.; Loi, M. A. Conjugated polymer-wrapped carbon nanotubes: Physical properties and device applications. Eur. Phys. J. B 2013, 86, 404.

[49] Lemasson, F.; Berton, N.; Tittmann, J.; Hennrich, F.; Kappes, M. M.; Mayor, M. Polymer library comprising fluorene and carbazole homo- and copolymers for selective single-walled carbon nanotubes extraction. Macromolecules 2012, 45, 713-722.

[50] Mistry, K. S.; Larsen, B. A.; Blackburn, J. L. High-yield dispersions of large-diameter semiconducting single-walled carbon nanotubes with tunable narrow chirality distributions. ACS Nano 2013, 7, 2231-2239.

[51] Falkovich, G. Fluid Mechanics: A Short Course for Physicists; Cambridge University Press: Cambridge, 2011.

[52] Morgan, H.; Green, N. G. AC Electrokinetics: Colloids and Nanoparticles; Research Studies Press: Baldock, UK, 2003.

[53] Saito, R.; Dresselhaus, G.; Dresselhaus, M. S.; Physical Properties of Carbon Nanotubes; Imperial College Press: London, 1998.

[54] Rao, R.; Liptak, D.; Cherukuri, T.; Yakobson, B. I.; Maruyama, B. In situ evidence for chirality-dependent growth rates of individual carbon nanotubes. Nat. Mater. 2012, 11, 213-216.

[55] Hida, T. Brownian Motion; Springer: New York, 1980; pp 44-113.

[56] Tsyboulski, D. A.; Bachilo, S. M.; Kolomeisky, A. B.; Weisman, R. B. Translational and rotational dynamics of individual single-walled carbon nanotubes in aqueous suspension. ACS Nano 2008, 2, 17701776.

[57] Broersma, S. Viscous force and torque constants for a cylinder. $J$. Chem. Phys. 1981, 74, 6989-6990.

[58] Han, Y.; Alsayed, A. M.; Nobili, M.; Zhang, J.; Lubensky, T. C.; Yodh, A. G. Brownian motion of an ellipsoid. Science 2006, 314, 626-630.

[59] Kim, Y.; Hong, S.; Jung, S.; Strano, M. S.; Choi, J.; Baik, S. Dielectrophoresis of surface conductance modulated single-walled carbon nanotubes using catanionic surfactants. J. Phys. Chem. B 2006, $110,1541-1545$.

[60] Li, W. S.; Hennrich, F.; Flavel, B. S.; Kappes, M. M.; Krupke, R. Chiral-Index resolved length mapping of carbon nanotubes in solution using electric-field induced differential absorption spectroscopy. Nanotechnology 2016, 27, 375706.

[61] Jones, T. B. Electromechanics of Particles; Cambridge University Press: Cambridge, 1995.

[62] Blatt, S.; Hennrich, F.; Löhneysen, H. V.; Kappes, M. M.; Vijayaraghavan, A.; Krupke, R. Influence of structural and dielectric anisotropy on the dielectrophoresis of single-walled carbon nanotubes. Nano Lett. 2007, 7, 1960-1966.

[63] Thomsen, C.; Reich, S.; Maultzsch, J. Carbon Nanotubes: Basic 
concepts and Physical Properties; Wiley-VCH Verlag GmbH, Weinheim, 2004.

[64] Xu, D. D.; Subramanian, A.; Dong, L. X.; Nelson, B. J. Shaping nanoelectrodes for high-precision dielectrophoretic assembly of carbon nanotubes. IEEE Trans. Nanotechnol. 2009, 8, 449-456.

[65] Small, E. W.; Isenberg, I. Hydrodynamic properties of a rigid molecule: Rotational and linear diffusion and fluorescence anisotropy. Biopolymers 1977, 16, 1907-1928.

[66] Ramos, A.; Morgan, H.; Green, N. G.; Castellanos, A. AC electrokinetics: A review of forces in microelectrode structures. J. Phys. D: Appl. Phys. 1998, 31, 2338-2353.

[67] Castellanos, A. Electrohydrodynamics; Springer: New York, 1998.

[68] Saville, D. A. Electrohydrodynamics: The taylor-melcher leaky dielectric model. Annu. Rev. Fluid Mech. 1997, 29, 27-64.

[69] Melcher, J. R.; Taylor, G. I. Electrohydrodynamics: A review of the role of interfacial shear stresses. Annu. Rev. Fluid Mech. 1969, $1,111-146$.

[70] Fetter, C. W. Contaminant Hydrogeology; Prentice Hall, New Jersey, 1999.

[71] Lide, D. R. CRC Handbook of Chemistry and Physics: A ReadyReference Book of Chemical and Physical Data; 90th ed. CRC: London, 2009.

[72] Baszkin, A.; Norde, W. Physical Chemistry of Biological Interfaces; CRC Press: Boca Raton, 1999.

[73] Burg, B. R.; Bianco, V.; Schneider, J.; Poulikakos, D. Electrokinetic framework of dielectrophoretic deposition devices. J. Appl. Phys. 2010, 107, 124308.

[74] Probstein, R. F. Physicochemical Hydrodynamics: An Introduction; 2nd ed. John Wiley \& Sons: New York, 1994.

[75] Kirby, B. J. Micro-and Nanoscale Fluid Mechanics: Transport in Microfluidic Devices; Cambridge University Press: Cambridge, 2010.

[76] Blatt, S. Dielectrophoresis of Single-Walled Carbon Nanotubes. Wissenschaftliche Berichte, FZKA-7431, Dissertation, Universität Karlsruhe, 2008. DOI: 10.5445/IR/200072592

[77] Kilic, M. S.; Bazant, M. Z.; Ajdari, A. Steric effects in the dynamics of electrolytes at large applied voltages. I. Double-layer charging. Phys. Rev. E 2007, 75, 021502.

[78] Israelachvili, J. N. Intermolecular and Surface Forces; 3rd ed. Academic Press: Amsterdam, 2011.

[79] Booth, F. Dielectric constant of polar liquids at high field strengths. J. Chem. Phys. 1955, 23, 453-457.

[80] Novikov, G. F.; Gapanovich, M. V.; Rabenok, E. V.; Bogdanova, L. M.; Kuzub, L. I. Dielectric properties of sols of silver nanoparticles capped by alkyl carboxylate ligands. Russ. Chem. Bull. 2011, 60, 419-425.

[81] Liu, H.; Qian, S. Z.; Bau, H. H. The effect of translocating cylindrical particles on the ionic current through a nanopore. Biophys. J. 2007, 92, 1164-1177.

[82] Kilic, M. S.; Bazant, M. Z.; Ajdari, A. Steric effects in the dynamics of electrolytes at large applied voltages. II. Modified Poisson-
Nernst-Planck equations. Phys. Rev. E 2007, 75, 021503.

[83] Paunov, V. N.; Dimova, R. I.; Kralchevsky, P. A.; Broze, G.; Mehreteab, A. The hydration repulsion between charged surfaces as an interplay of volume exclusion and dielectric saturation effects. J. Colloid Interface Sci. 1996, 182, 239-248.

[84] McClellan, A. L. Tables of Experimental Dipole Moments; Rahara Enterprises: El Cerrito, CA, 1974.

[85] Vijayaraghavan, A.; Blatt, S.; Weissenberger, D.; Oron-Carl, M.; Hennrich, F.; Gerthsen, D.; Hahn, H.; Krupke, R. Ultra-large-scale directed assembly of single-walled carbon nanotube devices. Nano Lett. 2007, 7, 1556-1560.

[86] Ritzoulis, G.; Papadopoulos, N.; Jannakoudakis, D. Densities, viscosities, and dielectric constants of acetonitrile+ toluene at 15,25, and 35.degree.C. J. Chem. Eng. Data 1986, 31, 146-148.

[87] Dunlap, W. C. Jr.; Watters, R. L. Direct measurement of the dielectric constants of silicon and germanium. Phys. Rev. 1953, 92, 1396-1397.

[88] Gray, P. R.; Hurst, P. J.; Lewis, S. H.; Meyer, R. G. Analysis and Design of Analog Integrated Circuits; 4th ed. Wiley: New York, 2001.

[89] Eranna, G. Crystal Growth and Evaluation of Silicon for VLSI and ULSI; CRC Press: Boca Raton, 2014.

[90] Srivastava, J. K.; Prasad, M.; Wagner, J. B. Jr. Electrical conductivity of silicon dioxide thermally grown on silicon. J. Electrochem. Soc. 1985, 132, 955-963.

[91] Burg, B. R.; Schneider, J.; Bianco, V.; Schirmer, N. C.; Poulikakos, D. Selective parallel integration of individual metallic single-walled carbon nanotubes from heterogeneous solutions. Langmuir 2010, 26, 10419-10424.

[92] Somasundaran, P.; Healy, T. W.; Fuerstenau, D. W. Surfactant adsorption at the solid-liquid interface-dependence of mechanism on chain length. J. Phys. Chem. 1964, 68, 3562-3566.

[93] Santos, F. J. V; De Castro, C. A. N.; Dymond, J. H.; Dalaouti, N. K.; Assael, M. J.; Nagashima, A. Standard reference data for the viscosity of toluene. J. Phys. Chem. Ref. Data 2006, 35, 1-8.

[94] de Castro, C. A. N.; Li, S. F. Y.; Nagashima, A.; Trengove, R. D.; Wakeham, W. A. Standard reference data for the thermal conductivity of liquids. J. Phys. Chem. Ref. Data 1986, 15, 1073-1086.

[95] Glen, N. F.; Johns, A. I. Determination of the density of toluene in the range from (293 to 373 ) K and from (0.1 to 30) MPa. J. Chem. Eng. Data 2009, 54, 2538-2545.

[96] Lide, D. R. CRC Handbook of Chemistry and Physics: A ReadyReference Book of Chemical and Physical Data; 85th ed. CRC Press: Boca Raton, 2004.

[97] O'Reilly, D. E.; Peterson, E. M. Self-diffusion coefficients and rotational correlation times in polar liquids. II. J. Chem. Phys. 1971, $55,2155-2163$.

[98] O'Reilly, D. E.; Peterson, E. M. Self-diffusion coefficients and rotational correlation times in polar liquids. III. Toluene. J. Chem. Phys. 1972, 56, 2262-2266.

[99] Atkins, P. W.; J. De Paula. Atkins' Physical Chemistry; 9th ed. Oxford University Press: Oxford, 2010. 